\title{
Highly efficient aqueous phase reduction of nitroarenes catalyzed by phosphine- decorated polymer immobilized ionic liquid stabilized PdNPs
}

Article

Accepted Version

Doherty, S., Knight, J. G., Backhouse, T., Bradford, A., Saunders, F., Bourne, R. A., Chamberlain, T. W., Stones, R., Clayton, A. and Lovelock, K. (2018) Highly efficient aqueous phase reduction of nitroarenes catalyzed by phosphinedecorated polymer immobilized ionic liquid stabilized PdNPs. Catalysis Science \& Technology, 8 (5). pp. 1454-1467. ISSN 2044-4761 doi: https://doi.org/10.1039/c7cy02557b Available at https://centaur.reading.ac.uk/75592/

It is advisable to refer to the publisher's version if you intend to cite from the work. See Guidance on citing.

To link to this article DOI: http://dx.doi.org/10.1039/c7cy02557b

Publisher: Royal Society of Chemistry

All outputs in CentAUR are protected by Intellectual Property Rights law, including copyright law. Copyright and IPR is retained by the creators or other copyright holders. Terms and conditions for use of this material are defined in the End User Agreement. 


\section{www.reading.ac.uk/centaur}

\section{CentAUR}

Central Archive at the University of Reading

Reading's research outputs online 


\section{Journal Name}

\section{ARTICLE}

\section{Highly efficient aqueous phase reduction of nitroarenes catalyzed by phosphine-decorated PEG-modified polymer immobilized ionic liquid stabilized PdNPs}

Received 00th January 20xx, Accepted 00th January 20xx

DOI: $10.1039 / \times 0 \times x 00000 x$

www.rsc.org/

\author{
S. Doherty, ${ }^{a,}{ }^{*}$ J. G. Knight,,${ }^{a}{ }^{*}$ T. Backhouse, ${ }^{a}$ A. Bradford, ${ }^{a}$ F. Saunders, ${ }^{a}$ R. A. Bourne, ${ }^{b, *}$ T. W. \\ Chamberlain, ${ }^{b,}{ }^{*}$ R. Stones, ${ }^{b}$ A. Clayton, ${ }^{b}$ and K. Lovelock ${ }^{c}$
}

\section{Introduction}

Aromatic amines are key motifs in a wide range of bioactive molecules and intermediates in the production of agrochemicals, pigments, dyes, polymers and additives. ${ }^{1}$ While there are a number of synthetic pathways available to this class of compound, including $\mathrm{N}$-arylation ${ }^{2}$ and catalytic reduction of imines or nitriles, ${ }^{3}$ the reduction of nitro compounds is perhaps the most versatile and widely used. ${ }^{4}$ Despite the popularity of this approach, there are a number of drawbacks including the need for either stoichiometric amounts of earth abundant metal reagent, ${ }^{5}$ often in combination with acid, high catalyst loadings ${ }^{6}$ and/or the use of

\footnotetext{
${ }^{a}$ NUCAT, School of Chemistry, Bedson Building, Newcastle University, Newcastle upon Tyne, NE1 TRU, UK.

E-mail: simon.doherty@ncl.ac.uk; Tel: +44 (0) 1912086537

${ }^{b}$ Institute of Process Research \& Development, School of Chemistry, University of Leeds, Woodhouse Lane, Leeds LS2 9JT, UK.

School of Chemistry, Food and Pharmacy, University of Reading, Reading, RG6 GAP, UK.

+ Electronic Supplementary Information (ESI) available: Synthesis and characterisation of imidazolium-based monomers, co-polymers $3 a-8 a, \mathrm{PdCl}_{4}$-loaded co-polymers $\mathbf{1 b} \mathbf{b} \mathbf{8} \boldsymbol{b}$ and polymer immobilised ionic liquid stabilised PdNPs $\mathbf{3 c - 8}$; TGA and DSC curves for $3 a-c$ to $8 a-c$, SEM images for $3 a-8 a, 3 b-8 b$ and $3 c-8 c$, TEM images of $1 c-8 c$, FTIR traces, $X$-ray photoelectron spectra for $3 b-8 b$ and $3 c-8 c$ and FD-XAS spectra for $\mathbf{1 b}$-c, $\mathbf{2 b}-\boldsymbol{c}, \mathbf{3} \boldsymbol{b}$-c and $\mathbf{4 b}$-c. See DOI: 10.1039/x0xx00000x
}

toxic reducing agents. ${ }^{7}$ In addition, harsh reaction conditions, the use of harmful organic solvent, poor functional group tolerance, partial reduction to hydroxylamines, hydrazones, azoarenes and azoxyarenes and contamination of the product with metal all limit the potential applications of this technology. As such there is considerable interest in developing selective catalysts that operate under mild conditions at low catalyst loadings in environmentally green solvents.

In this regard, metal nanoparticles stabilized by either inorganic or carbon-based materials are evolving into an efficient class of catalyst for the reduction of aromatic nitro compounds as they have been shown to have high activity and selectivity and allow for recycling and scale-up using continuous flow technology. ${ }^{8}$ Examples of systems with encouraging reaction credentials for either hydrogenation or transfer hydrogenation of nitroarenes include palladium nanoparticles supported on aminofunctionalized mesocellular foam, ${ }^{9}$ size-controlled palladium nanoparticles immobilized on carbon nanospheres, ${ }^{10}$ PVPstabilized palladium nanoparticles, ${ }^{11}$ magnetically separable $\mathrm{Fe}-\mathrm{Ni}$ bimetallic nanoparticles ${ }^{12}$ and gold nanoparticles supported by imidazolium-based porous organic polymers. ${ }^{13}$ Other recently developed systems with promising performance profiles include palladium nanoparticles stabilized by polymethylhydrosiloxane, ${ }^{14}$ $\mathrm{Nb}_{2} \mathrm{O}_{5}$ nanowire, ${ }^{15}$ amine-functionalized hollow magnetic 
nanoparticles, ${ }^{16} \mathrm{Fe}_{3} \mathrm{O}_{4}$ magnetic nanocomposites combined with graphene oxide and carbon nanotubes, ${ }^{17}$ phosphinefunctionalized ionic liquids, ${ }^{18}$ multi-walled carbon nanotubes, ${ }^{19}$ nitrogen functionalized active carbon ${ }^{20}$ and gold nanoparticles supported by imidazolium based porous organic polymers or graphene oxide/carbon nanotubes. ${ }^{21}$ While some of these systems possess desirable reaction credentials, very few combine all the criteria that are considered essential for an environmentally green and sustainable process, i.e. low catalyst loadings, short reaction times, operationally straightforward protocols, high selectivity, elimination of organic solvent, efficient recyclability and mild conditions. Thus, there is immense potential and opportunity to develop new catalyst technology to address each of these issues and identify improved catalysts. One such system based on $\mathrm{Fe} / \mathrm{ppm} \mathrm{Pd}$ nanoparticles and a PEG-containing designer surfactant appears to meet the majority of these criteria by delivering safe, sustainable, environmentally responsible and selective reduction of nitro groups in water at room temperature. ${ }^{22}$

Ionic liquids are an intriguing class of solvent that has also been widely used in catalysis and more recently as both solvent and stabilizer for transition metal nanoparticles. ${ }^{23}$ However, while this stabilization is believed to result from weak electrostatic interactions that are easily displaced to generate the active site, they are often not sufficient to prevent nanoparticle aggregation under the conditions of catalysis. ${ }^{24}$ To this end, metal binding heteroatom donors have been incorporated into ionic liquids on the basis that the heteroatom would bind to the nanoparticle and supplement the weak electrostatic interactions and provide long term stability for use in catalysis. ${ }^{25}$ Although this strategy has been successfully applied in a number of cases, ${ }^{26}$ the large volumes required for catalysis and leaching during work-up are major issues that will hamper their implementation and applications.

We have been exploring the concept of Polymer Immobilized Ionic Liquid Phase (PIILP) catalysis ${ }^{27}$ to combine favourable characteristics of ionic liquids, such as their tunable physicochemical properties and functionalization, with the advantages of attachment to a solid support, which will limit loss of ionic liquid, facilitate separation and recovery and reduce the volume of ionic liquid as the catalyst would be retained in a small amount of polymer immobilized ionic liquid. In addition to improving the long term stability and recyclability of the nanoparticles, incorporation of a heteroatom donor into a polymer immobilized ionic liquid presents a number of additional benefits including modification of the electronic structure of the metal surface, control of nanoparticle size and morphology and the capacity to tune the hydrophilicity of the ligand environment. ${ }^{28}$ Gratifyingly, our initial foray in this area demonstrated that PEG-modified phosphine-decorated polymer immobilized ionic liquid stabilized palladium nanoparticles (PdNP@PPh 2 -PEGPIILP) are remarkably active and selective catalysts for the aqueous phase hydrogenation of $\alpha, \beta$-unsaturated aldehydes, ketones, amides, esters and nitriles giving high conversions and up to $100 \%$ selectivity for reduction of the $\mathrm{C}=\mathrm{C}$ double bond. ${ }^{29}$ Interestingly, both the heteroatom donor phosphine and the immobilized ionic liquid appear to be essential to achieving the high activity and selectivity. With the aim of further exploring the efficacy of PdNP@PIILP-based systems and investigating whether the same features determine their performance as catalysts in other reductions, we extended our studies to include a comparative study of the aqueous phase hydrogenation and transfer hydrogenation of nitroarenes. This study also aimed to investigate the effect on catalyst properties/performance of introducing three-fold cross-linking in the form of tris( $p$-vinylphenyl) phosphine ${ }^{30}$ on the basis that it should isolate the heteroatom donors and thereby influence the number and type of metal-donor interactions; in this manner we hoped to obtain a more robust catalyst as well as demonstrate whether $\mathrm{Pd}$--- $\mathrm{PPh}_{2}$ interactions control NP formation. In this regard, Yuan recently demonstrated that the size, size distribution and stability of a range of metal clusters could be controlled by an unusual synergy between the 'conventional' stabilization provided by heterocyclic cations in polyionic liquids and an in situ generated polycarbene; a record high for the catalytic performance in the methanolysis of ammonia borane was reported. ${ }^{31}$ Thus, it would be eminently reasonable to expect this mechanism of metal cluster stabilization to translate to the phosphine-decorated polymer immobilized ionic liquids described herein. The polymers developed in this project and the associated nomenclature designed to identify their composition are shown in Figure 1.

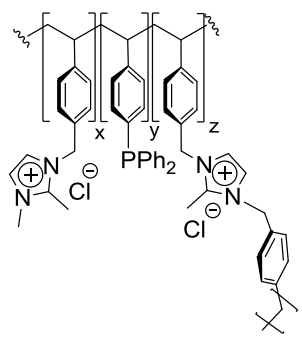

$\mathrm{PPh}_{2}-\mathrm{PIILP} 1 \mathrm{a}$

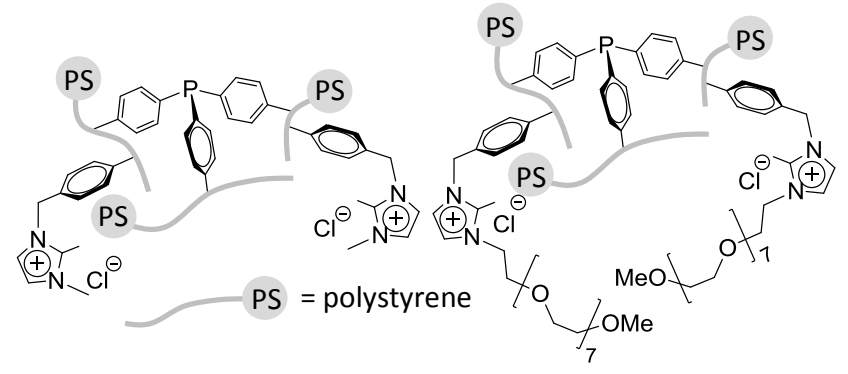

PSty $_{3}$-PIILP 3a

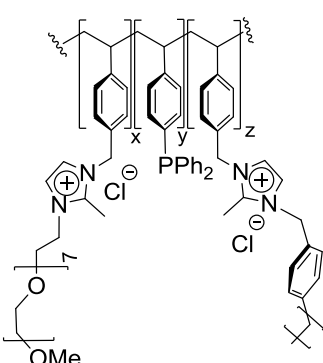

$\mathrm{PPh}_{2}$-PEGPIILP 2a

$$
\begin{aligned}
& \text { Summary of } \mathrm{Pd}((\mathrm{I}) \text { and PdNP-based PIILs } \\
& \mathrm{PdCl}_{4} @ P P h_{2}-\mathrm{PIILP}, 1 \mathbf{b} ; \mathrm{PdNP} @ P P h_{2}-\mathrm{PIILP}, 1 \mathbf{c} \\
& \mathrm{PdCl}_{4} @ P P h_{2}-P E G P I I L P, 2 b ; P d N P @ P P h_{2}-P E G P I I L P, 2 c \\
& \mathrm{PdCl}_{4} @ P S t y \text {-PIILP, 3b; PdNP@PSty }- \text { PIILP, 3c } \\
& \mathrm{PdCl}_{4} @ P S t y_{3}-\mathrm{PEGPIILP}, 4 \mathbf{b} ; \text { PdNP@PSty } 3 \text {-PEGPIILP, 4c }
\end{aligned}
$$

Fig 1. Composition and formulation of polymer immobilized ionic liquids 1a-4a and their $\left[\mathrm{PdCl}_{4}\right]^{2-}$ loaded counterparts (1b-4b) and PdNPs (1c-4c).

While PdNPs stabilized by ionic liquids immobilized on either a porous polymer ${ }^{32}$ or a support such as graphene oxide-based 
nanocomposites, ${ }^{33}$ magnetic core shell nanoparticles ${ }^{26 r}$ or silica ${ }^{34}$ catalyze the reduction of nitroarenes, the majority of these systems suffer limitations such as the need for high catalyst loadings, elevated reaction temperatures or long reaction times, the use of organic solvents or a large excess of reducing agent. Herein, we report that PdNPs stabilized by PEG-modified phosphine-decorated polymer-immobilized ionic liquids are remarkably efficient and selective catalysts for the aqueous phase hydrogenation of nitroarenes under exceptionally mild conditions as well as $\mathrm{NaBH}_{4}$-mediated hydrogenations in batch and continuous flow. Tc 'his end, while the stabilization of palladium nanoparticles for use in aqueous phase catalysis is a burgeoning area of interest there are surprisingly few reports of the reduction of nitroarenes in water and, to the best of our knowledge, the optimum system described herein is one of the most efficient to be reported for this class of substrate, even though reactions occur under diffusion control. Indeed, the newly developed catalyst operates with high efficiency at ambient temperature and gives excellent yields in short reaction times with a low catalyst loading, either at low hydrogenation pressure or with only a slight excess of reducing agent. Moreover, the same system also catalyzes a tandem aqueous phase Suzuki-Miyaura cross-couplingnitroarene reduction sequence to afford high yields of biaryl amine in an operationally straightforward single-pot protocol.

\section{Results and Discussi-n}

\section{Synthesis and Characterization of Heteroatom-Donor Stabilized} Palladium Nanoparticles

Polymers 1a and $\mathbf{2 a}$ were prepared as previously described as were precursors $\mathrm{PdCl}_{4} @ \mathrm{PPh}_{2}-\mathrm{PIILP}(\mathbf{1 b})$ and $\mathrm{PdCl}_{4} @ \mathrm{PPh}_{2}-\mathrm{PEGPIILP}$ (2b) and their corresponding PIILP-stabilized PdNPs, 1c and 2c, respectively. ${ }^{29}$ Threefold phosphine cross-linked PIILP $\mathbf{3 a}$ was prepared by AIBN initiated radical polymerization of the corresponding imidazolium monomer with $\mathrm{P}(4 \text {-vinylphenyl })_{3}$ in a 1:1 mixture of THF/ethanol at $80^{\circ} \mathrm{C}$ and its PEGylated counterpart 4a was prepared in a similar manner from $\mathrm{P}\left(4\right.$-vinylphenyl) ${ }_{3}$ and 2 methyl-1-(2,5,8,11,14,17,20,23-octaoxapentacosan-25-yl)-3-(4vinylbenzyl)- $1 \mathrm{H}-3 \lambda 4$-imidazolium chloride with the aim of increasing hydrophilicity and water solubility/compatibility for use in aqueous phase catalysis. As for $\mathbf{1 a}$ and $\mathbf{2 a}$, a 1:2 ratio of $\mathrm{P}(4-$ vinylphenyl $)_{3}$ to imidazolium monomer was retained to ensure that complete exchange of halide for the tetrachloropalladate anion would result in a palladium to phosphine ratio of one; this would then enable a direct and meaningful comparison with our earlier systems. The corresponding PIIL-stabilized PdNPs were prepared by sodium borohydride reduction of $\mathbf{3 b}$ and $\mathbf{4 b}$, respectively, and isolated as black powders in good yield. Polymers 5a-9a (vide infra) were designed to examine the influence on catalyst performance of the polymer components polyethyleneglycol (PEG), ionic liquid and phosphine and were prepared from the constituent monomers according to the procedure described for 1a-4a. Full details of the synthesis and compound characterization data are provided in the ESI.

The solid state ${ }^{31} \mathrm{P}$ NMR spectra of $\mathrm{PdCl}_{4} @ \mathrm{PPh}_{2}-\mathrm{PIILP}$ (1) $\mathrm{PdCl}_{4} @ \mathrm{PPh}_{2}$-PEGPIILP (2b), PdNP@PPh - PIILP (1c) and $P d N P @ P P h_{2}-P E G P I I L P \quad(2 c)$ confirm the presence of $P d---P$ interactions, which are clearly apparent from the low-field chemical shifts of $\delta 26.8,27.9,29.5$ and 28.8 ppm, respectively; these are similar to previously reported data. ${ }^{35}$ In addition, the absence of any high-field signals in the region of $\delta-5$ to $-7 \mathrm{ppm}$ indicates that there is no uncoordinated $\mathrm{PPh}_{2}$. In stark contrast, the corresponding spectra for $\mathbf{3 b} \mathbf{b} \mathbf{- 4} \mathbf{b}$ and $\mathbf{3 c}-\mathbf{4} \mathbf{c}$ reveal that a small fraction $(<10 \%)$ of the phosphine remains uncoordinated as evidenced by minor signals at $\delta-4.1$ and $-4.8 \mathrm{ppm}$, respectively (ESI, Figures S36, S41, S64 and S70). Surface characterization of the palladium in $\mathbf{1 c}-\mathbf{4 c}$ was undertaken with $\mathrm{X}$-ray photoelectron sper+roscopy (XPS) by analysis of the $\mathrm{Pd} 3 \mathrm{~d}_{3 / 2}$ and $\mathrm{Pd} 3 \mathrm{~d}_{5 / 2}$ doublets (Figure 2a-d). The peaks with binding energies (BE)

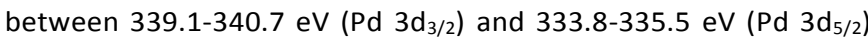
are attributed to $\mathrm{Pd}(0)$ species while those with binding energies

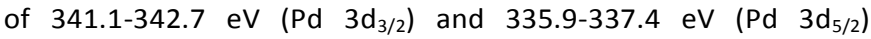
correspond to $\mathrm{Pd}(\mathrm{II})$ species; the latter most likely results from reoxidation of $\operatorname{Pd}(0)$. The $\mathrm{X}$-ray photoelectron spectra of $\mathbf{1} \mathbf{b}-\mathbf{4 b}$ contain characteristic Pd $3 \mathrm{~d}$ doublets with binding energies of 336.6-337.5 eV and 341.8-342.8 eV, which is consistent with the presence of $\mathrm{Pd}(\mathrm{II})$ associated with phosphine coordinated chloropalladate. Together, the solid state NMR data and palladium binding energies $\left(3 d_{5 / 2}\right.$ and $\left.3 d_{3 / 2}\right)$ are consistent with the phosphine providing the primary stabilization of the nanoparticles; this is in agreement with data reported for other phosphinestabilized palladium nanoparticles. ${ }^{26 q, 36}$ (a)

(c)
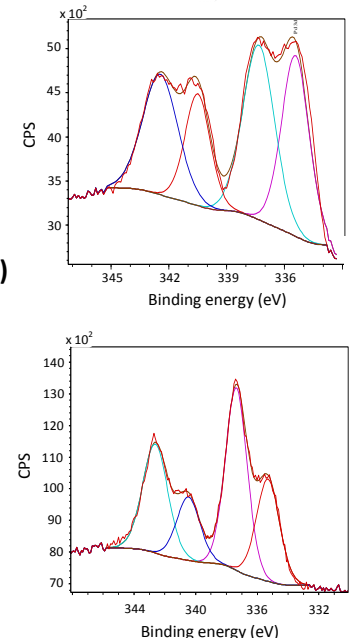

(b)
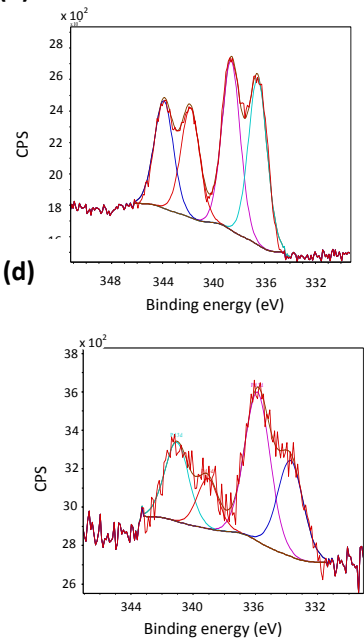

Fig. 2 Pd 3d core level XPS spectrum of 1c-4c (a-d respectively) referenced to the hydrocarbon C 1s. X-ray source: monochromatic Al K $\alpha$.

Fluorescence-detected X-ray absorption spectroscopy (FD XAS) showed a general increase in the Pd LIII edge energy relative to the Pd precursor, $\mathrm{Na}_{2}\left[\mathrm{PdCl}_{4}\right]$, and $\mathrm{Pd} / \mathrm{C}$ controls for $\mathbf{1 b}-\mathbf{4 b}$ and $\mathbf{1 c}-$ 4c materials, respectively, indicating a small reduction of the electron density on the palladium metal within the polymer supports (ESI, Figures S103-S106). However, there is a significantly smaller change in white line intensity for catalyst $\mathbf{2 c}$ and $\mathbf{4 c}$ upon reduction compared to $\mathbf{1 c}$ and $\mathbf{3 c}$, implying that the addition of the PEG to the polymer support influences the amount of electron 
density located on the palladium metal in the final catalyst materials. This observation is slightly at odds with the XPS data, which might be related to surface sensitive nature of XPS, however, this requires further investigation. Thermogravimetric analysis of 1a-4a shows that these polymers are stable up to 225$300{ }^{\circ} \mathrm{C}$ (ESI, Figures S6 and S11) and the initial weight loss before the onset of decomposition is attributed to removal of absorbed water molecules trapped in the ionic environment. These TGA profiles confirm that 1a-4a should be ideally suited for use as catalyst supports even under harsh reactions conditions.
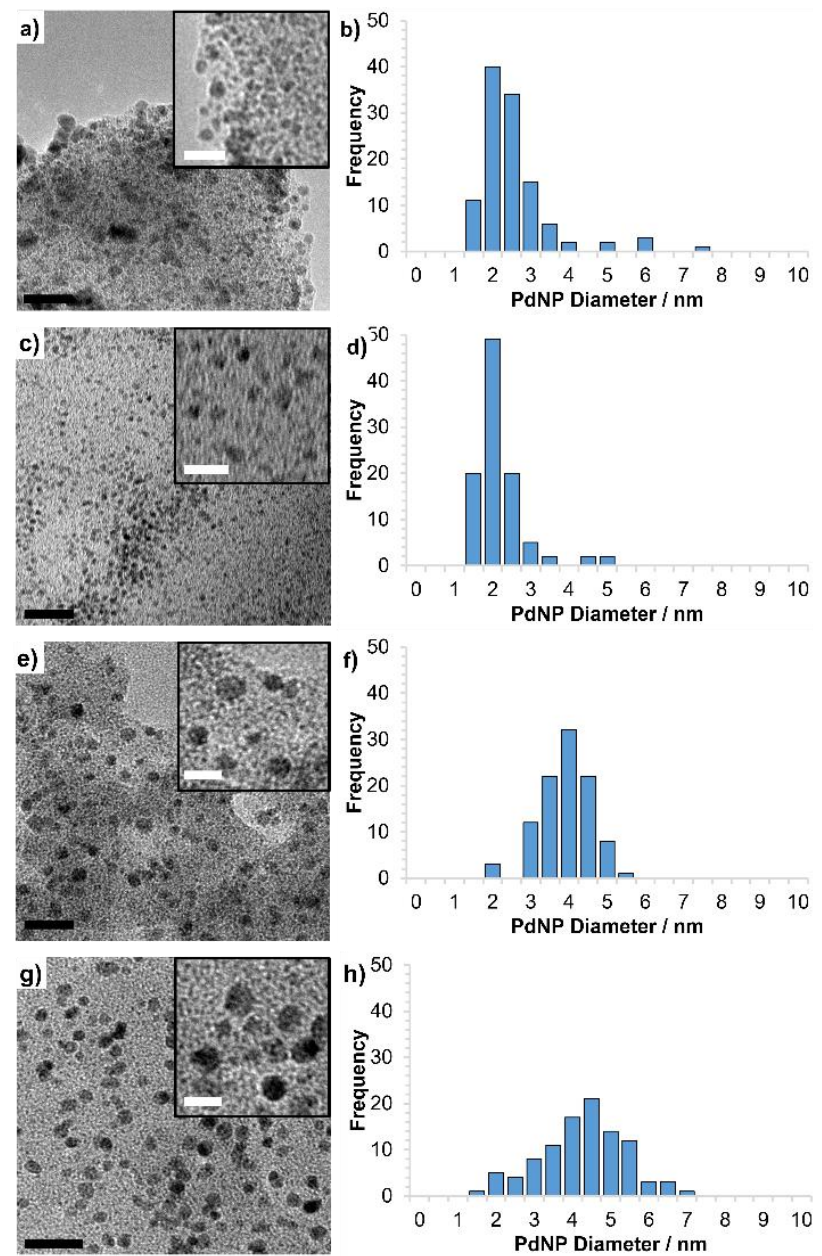

Fig. 3 HRTEM images of (a) PdNP@PIILP (1c), (c) PdNP@PPh ${ }_{2}-P E G P I I L P(2 c),(e)$ PdNP@PSty ${ }_{3}$-PIILP (3c) and (g) PdNP@PSty ${ }_{3}$-PEGPIILP (4c) and (b, d, f and h) corresponding particle size distributions determined by counting $>100$ particles. Mean nanoparticle diameters are $2.29 \pm 0.96 \mathrm{~nm}, 1.93 \pm 0.67 \mathrm{~nm}, 3.67 \pm 0.67 \mathrm{~nm}$ and $4.02 \pm 1.11 \mathrm{~nm}$ for $\mathbf{1 c}-\mathbf{4 c}$ respectively. Black and white scale bars are 5 and $10 \mathrm{~nm}$ respectively.

TEM micrographs revealed that $\mathbf{1 c}$ and $\mathbf{2 c}$ consist of small, near monodisperse, nanoparticles with average diameters of $2.29 \pm$ 0.96 and $1.93 \pm 0.67 \mathrm{~nm}$, respectively, while the nanoparticles in the highly cross-linked systems $\mathbf{3 c}$ and $\mathbf{4 c}$ are larger with average diameters of $3.67 \pm 0.67$ and $4.01 \pm 1.11 \mathrm{~nm}$, respectively; the micrographs and associated distribution histograms based on > 100 particles are shown in Figure 3 . Interestingly, at this stage it appears that site isolation of the heteroatom by extensive cross-linking with tris(4-vinylphenyl)phosphine has a marked influence on particle size and distribution as the palladium to phosphine ratio in each of these systems is the same by virtue of the 2 to 1 ratio of phosphine to imidazolium-based monomer. Further studies are currently underway to vary the palladium to phosphine ratio as well as the extent of site isolation in order to explore and quantify how the Pd----P interactions in the palladatebased precursor influence the size and distribution of the nanoparticles and the kinetics of formation. As a comparison, palladium nanoparticles with mean diameters between $2.7 \mathrm{~nm}$ and $3.6 \mathrm{~nm}$ have recently been prepared by reduction of $[\mathrm{Pd}(\mathrm{acac})(\mathrm{COD})]\left[\mathrm{BF}_{4}\right]$ and $\mathrm{Pd}(\mathrm{acac})_{2}$ in the presence of phosphinefunctionalized ionophilic ligands while in the absence of ligand aggregates formed. ${ }^{26 p, q}$

\section{Hydrogenation of Nitroarenes}

Preliminary optimizations were conducted in a stirred bench-top reactor under mild conditions $\left(25^{\circ} \mathrm{C}\right)$ using a $0.47 \mathrm{~mol} \%$ loading of $2 c$ to examine the effect of catalyst, solvent and pressure on conversion and selectivity, full details are presented in Table 1 and Figure 4. A survey of solvent showed that high conversions were obtained in water and water/ethanol while reactions conducted in conventional organic solvents gave much lower conversions (entries 1-6). The practical advantages associated with aqueous phase catalysis together with the environmentally benign properties of water and the potential benefits of the 'hydrophobic effect' prompted us to perform the remainder of our studies in this solvent.

Table 1 Hydrogenation of nitrobenzene as a function of catalyst and solvent. ${ }^{a}$

\begin{tabular}{|c|c|c|c|c|}
\hline Catalyst & Solvent & Time (min) & $\% Y^{\prime}{ }^{2} d^{b}(d)^{c}$ & $\operatorname{TOF}\left(\mathrm{h}^{-1}\right)^{d}$ \\
\hline $2 c$ & water & 90 & $100(-)$ & $<142$ \\
\hline $2 c$ & water/EtOH & 90 & $99(1)$ & 140 \\
\hline 2c & ethanol & 90 & $83(5)$ & 117 \\
\hline $2 c$ & ethyl acetate & 90 & $18(2)$ & 26 \\
\hline 2c & toluene & 90 & $5(2)$ & 7 \\
\hline $2 c$ & $\mathrm{MeCN}$ & 90 & $7(2)$ & 10 \\
\hline $\mathrm{Pd} / \mathrm{C}$ & Watere & 90 & $61(4)$ & 86 \\
\hline $1 c$ & water & 90 & $60(2)$ & 35 \\
\hline $3 c$ & water & 90 & $81(3)$ & 120 \\
\hline $4 c$ & water & 90 & $72(4)$ & 59 \\
\hline
\end{tabular}

${ }^{a}$ Reaction conditions: $1.0 \mathrm{mmol}$ nitrobenzene, mol\% Pd in 1c $(0.89 \mathrm{~mol} \%), 2 \mathrm{c}$ (0.47 mol\%), 3c (0.45 mol\%), 4c (0.81 mol\%), $10 \mathrm{~mL}$ solvent, $25{ }^{\circ} \mathrm{C}, 70 \mathrm{psi} \mathrm{H}_{2}$ pressure, time. ${ }^{b}$ Yields determined by ${ }^{1} \mathrm{H}$ NMR spectroscopy using dioxane as internal standard and gas chromatography using decane as internal standard. Average of three runs. ${ }^{c}$ average deviation $(d)=\left(\left|x_{1}-\bar{x}\right|+\left|\mathrm{x}_{2}-\bar{x}\right|+\left|\mathrm{x}_{3}+\bar{x}\right|\right) / 3$ where $\bar{x}=\left(\mathrm{x}_{1}+\mathrm{x}_{2}+\mathrm{x}_{3}\right) / 3$ and $\mathrm{x}_{\mathrm{n}}$ is the conversion for run $\mathrm{n} .{ }^{d}$ Moles product per mole catalyst per hour based on total palladium content. e $1.0 \mathrm{mmol}$ nitrobenzene, $0.47 \mathrm{~mol} \% \mathrm{Pd} / \mathrm{C}, 70 \mathrm{psi} \mathrm{H}_{2}, 25^{\circ} \mathrm{C}, 10 \mathrm{~mL}$ water. 


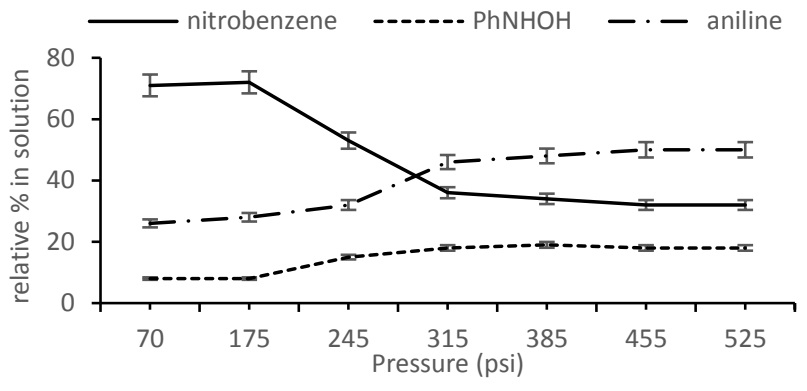

Fig. 4 Reaction profile as a function of pressure for the hydrogenation of nitrobenzene Reaction conditions: $1.0 \mathrm{mmol}$ nitrobenzene, mol\% Pd in $2 \mathrm{c}(0.47 \mathrm{~mol} \%), 10 \mathrm{~mL}$ water, $30 \mathrm{~min}$ at $25^{\circ} \mathrm{C}$.

A study of the variation in conversion/composition as a function of pressure for the hydrogenation of nitrobenzene catalysed by $\mathbf{2 c}$ in water at room temperature for 30 min showed that conversions increased with increasing pressure up to $68 \%$ at 315 psi (Figure 4). While this profile is a clear indication of mass transfer limited dissolution of hydrogen in water the reaction will most likely still be under diffusion control, even under a high pressure of hydrogen, due to the poor solubility of the substrate. In addition, the short reaction time required for this study revealed the presence of varying quantities of hydroxylamine as the only observable intermediate, which suggests that reduction of this species is slow; however, full conversion to aniline as the sole product was obtained when the reaction time was extended to $90 \mathrm{~min}$. A reduction in the catalyst loading to $0.047 \mathrm{~mol} \%$ also resulted in a significant increase in activity such that conversion of $15 \%$ and $24 \%$ were obtained at 70 psi and 315 psi of hydrogen, respectively, after only $30 \mathrm{~min}$ at room temperature; this corresponds to TOFs of $580 \mathrm{~h}^{-1}$ and $1021 \mathrm{~h}^{-1}$, respectively, (measured as moles of product per mole of catalyst). However, as high conversions could be obtained at room temperature under a hydrogen pressure as low as $70 \mathrm{psi}$, all further reactions were conducted at this pressure by extending the reaction time where necessary. Under these conditions, comparative catalyst testing of 1c- $\mathbf{4} \mathbf{c}$ revealed that modification by removal of the PEG resulted in a marked reduction in performance as 1c only gave $60 \%$ conversion in the same time (entry 8 ), while the introduction of the phosphine as a cross-linker also resulted in a slight reduction in activity as 3c and $4 \mathrm{c}$ gave conversions of $81 \%$ and $72 \%$, respectively (entries 9-10). For comparison, $0.47 \mathrm{~mol} \% \mathrm{Pd} / \mathrm{C}$ catalysed this hydrogenation under the same conditions but only reached $61 \%$ conversion after $1.5 \mathrm{~h}$ (entry 7 ). The efficacy of $\mathbf{2 c}$ was further tested by reducing the catalyst loading even further to $0.0018 \mathrm{~mol} \%$ and under otherwise identical conditions the conversion of $65 \%$ after $15 \mathrm{~h}$ corresponds to a total turnover number (TON, measured as total moles of product per mole of catalyst) of 36,100 and a TOF of $2,400 \mathrm{~h}^{-1}$. This is probably more representative of the potential intrinsic turnover rate of the catalyst, particularly as a further decrease in catalyst loading to 0.001 mol\% resulted in a marginal increase in TON to 37,300 , in the same time. The efficacy of $\mathbf{2 c}$ compared with $\mathbf{1 c}$ is most likely associated with the hydrophilicity of the PEGylated support increasing the dispersibility of the catalyst and thereby facilitating access of the substrate to the active site.
A survey of the relevant literature reveals that even though these reactions occur under diffusion control $2 \mathrm{c}$ outperforms existing systems and, in the majority of cases, by quite some margin. ${ }^{17,19,20,32,34}$ For example, the TOF of $1021 \mathrm{~h}^{-1}$ obtained with $2 \mathrm{c}$ in water at room temperature under 315 psi of hydrogen is significantly higher than $499 \mathrm{~h}^{-1}$ for an assembly of $\mathrm{PdNPs}^{\text {in }} \mathrm{SiO}_{2}-$ supported ionic liquid brushes, ${ }^{34}$ and is a substantial improvement on that of $30 \mathrm{~h}^{-1}$ for PdNPs supported on graphene oxide/carbon nanotubes- $\mathrm{Fe}_{3} \mathrm{O}_{4}$ at $60{ }^{\circ} \mathrm{C}_{1}^{17} 50 \mathrm{~h}^{-1}$ for PdNPs immobilized in porous imidazolium-based ionic liquid polymers at $50{ }^{\circ} \mathrm{C}, 3030 \mathrm{~h}^{-1}$ for Pd-Pt bimetallic nanoparticles on functionalized multi-wallcarbon nanotubes ${ }^{19}$ and $130 \mathrm{~h}^{-1}$ for PdNPs supported on nitrogen functionalized active carbon. ${ }^{20}$ Good conversions have also been achieved with a range of other PdNP-based systems but reactions were typically conducted in organic solvents such as methanol, ethanol, toluene, ionic liquid or ethyl acetate and often required elevated temperatures which precludes a viable comparison; even so the TOFs obtained with these systems are typically lower than those for 2c. ${ }^{5 b, 9 a, 16,18,37}$

The conditions identified above have also been applied to the hydrogenation of a range of substituted aromatic and heteroaromatic nitro compounds to assess the scope and efficacy of 2c (Table 2). Good conversions were obtained for nitroarenes substituted with electron-donating groups such as methoxy, hydroxy and methyl (entries 1-3) and electron withdrawing groups such as ester, cyano and ketone, all of which were reduced to the corresponding aniline at room temperature in reasonably short reaction times (entries $3-6$ ). Catalyst $\mathbf{2 c}$ is functional group tolerant and highly chemoselective as substrates bearing reducible substituents such as cyano, ester and ketone all gave the corresponding amine as the sole product (entries 4-6). Surprisingly, hydrogenation of 4-chloro-1-nitrobenzene and 2chloro-1-nitrobenzene resulted in efficient hydrodechlorination to afford aniline as the sole product in $98 \%$ and $99 \%$ yield, respectively, after $12 \mathrm{~h}$ (entries 7-8). Such an efficient and selective hydrodechlorination is quite remarkable and has not previously been documented and studies are currently underway to elucidate the pathway for this process and to establish what factors influence selectivity. In this regard, hydrodehalogenation is a common competing reaction during the hydrogenation of halosubstituted nitroarenes catalysed by palladium nanoparticles but in such cases aniline is generally a relatively minor by-product. ${ }^{38}$ Sterically hindered substrates such as 2-substituted nitrobenzenes also gave good conversions at room temperature and in reasonably short reaction times (entries 9-11) with the exception of 1-nitronaphthalene which required significantly longer but eventually reached $73 \%$ conversion after $16 \mathrm{~h}$ (entry 12 ). The same protocol was extended to the reduction of heterocyclic nitroarenes including 2- and 3-nitropyridine and 6- and 8nitroquinoline which gave the corresponding amines in high yield after short reaction times (entries 13-16). In stark contrast, 4nitroindole proved to be a much more challenging substrate and only reached $27 \%$ conversion after $150 \mathrm{~min}$, however, practical conversions could be obtained by extending the reaction time accordingly. Finally, 6-nitrobenzothiazole proved to be completely resistant to hydrogenation by $\mathbf{2 c}$ as the substrate was recovered in 
quantitative yield even after a reaction time of $16 \mathrm{~h}$. At this stage, we suggest that benzothiazole poisons or inhibits the

Table 2 Hydrogenation of aromatic and heteroaromatic nitroarenes catalysed by PdNP@PPh 2 -PEGPIILP (2c) ${ }^{a}$

water/ethanol

a Reaction conditions: $1 \mathrm{mmol}$ nitrobenzene, $0.47 \mathrm{~mol} \% 2 \mathrm{c}, 10 \mathrm{~mL}$ solvent, $25{ }^{\circ} \mathrm{C}$ $\mathrm{H}_{2}$ pressure 70 psi, time. ${ }^{b}$ Yields determined by ${ }^{1} \mathrm{H}$ NMR spectroscopy using dioxane as internal standard and gas chromatography using decane as internal standard. Average of at least three runs. ${ }^{c}$ average deviation $(d)=\left(\left|\mathrm{x}_{1}-\bar{x}\right|+\mid \mathrm{x}_{2}\right.$ $\left.-\bar{x}|+| \mathrm{x}_{3}+\bar{x} \mid\right) / 3$ where $\bar{x}=\left(\mathrm{x}_{1}+\mathrm{x}_{2}+\mathrm{x}_{3}\right) / 3$ and $\mathrm{x}_{\mathrm{n}}$ is the conversion for run $\mathrm{n}{ }^{d}$ Yield of aniline resulting from complete hydrodechlorination.

catalyst by coordination of the sulfur donor atom to the surface of the nanoparticle, on the basis that a competition reaction containing 6-nitrobenzothiazole and nitrobenzene catalysed by $\mathbf{2 c}$ only gave $7 \%$ conversion to aniline after 90 min, compared with the quantitative conversion obtained in the absence of 6-nitrobenzothiazole. Rather surprisingly, even though a number of the substrates in Table 2 are solids and either poorly soluble or insoluble in water, good yields of amine were obtained despite the fact that dissolution will limit reaction efficiency.

The influence on catalyst performance of the surface ionic liquid, phosphine and PEG components has been systematically examined by comparing the performance of PdNP@PPh 2 -PEGPIILP (2c) against PdNP@PPh ${ }_{2}-$ PIILP (1c), PdNP@PPh ${ }_{2}-$ PEGstyrene (5c) and PdNP@PEGPIILP (6c) under the standard conditions described above (See Table S1 in the ESI for structural details). The data in Table 3 shows that selective removal of PEG results in a decrease in TOF from 142 $\mathrm{h}^{-1}$ for $\mathbf{2 c}$ to $43 \mathrm{~h}^{-1}$ for $\mathbf{1 c}$, which most probably reflects an improvement in dispersibility for the PEG-based system in water, particularly since the mean diameters of the nanoparticles in both systems are similar. Interestingly selective removal of the $\mathrm{PPh}_{2}$ also resulted in a significant drop in activity to $44 \mathrm{~h}^{-1}$ for ionic liquid-PEG based $\mathbf{6 c}$. Since a PEGionic liquid based system is expected to be highly dispersed and water soluble the drop in activity may well be associated with the larger size of the NPs in $6 c(3.23 \pm 0.61 \mathrm{~nm})$ compared with $2 c(1.93 \pm 0.67 \mathrm{~nm})$. Finally, selective removal of the ionic liquid component also results in a drop in TOF to $69 \mathrm{~h}^{-1}$ for $5 \mathrm{c}$; this may also reflect the water solubilizing effect of the ionic liquid component as the mean diameter of the NPs in $\mathbf{5 c}$ $(1.83 \pm 0.44 \mathrm{~nm})$ are similar to that for $\mathbf{2 c}$. Although it is clear that each component has a direct and dramatic effect on catalyst performance further studies will be required to establish the role of the heteroatom donor i.e. whether it influences NP formation and size and/or surface properties or modifies catalyst dispersibility and solubility. Similarly, the efficiency of catalysts with combinations of two components removed have also been examined, namely $\mathrm{PPh}_{2}$-styrene copolymer, PdNP@PPh ${ }_{2}$-styrene (7c) and imidazolium-based polyionic liquid PdNP@PIILP (8c) and both were shown to be markedly less efficient than $\mathbf{2 c}$ under the same conditions.

Table 3 Hydrogenation of nitrobenzene as a function of catalyst composition ${ }^{a}$

\begin{tabular}{cccc}
\hline Catalyst & Yield $(\%) /$ TOF $\left(\mathrm{h}^{-1}\right)^{b, c}$ & Catalyst & \% Yield/TOF $\left(\mathrm{h}^{-1}\right)^{b, c}$ \\
\hline 1c & $38 / 43$ & 5c & $34 / 69$ \\
2c & $66 / 140$ & 6c & $38 / 44$ \\
3c & $55 / 122$ & 7c & $21 / 62$ \\
4c & $45 / 56$ & 8c & $44 / 23$
\end{tabular}

${ }^{a}$ Reaction conditions: $1 \mathrm{mmol}$ nitrobenzene, catalyst $1 \mathrm{c}-8 \mathrm{c}, 10 \mathrm{~mL}$ water, $25^{\circ} \mathrm{C}$ 70 psi $\mathrm{H}_{2}, 60$ min. ${ }^{b}$ Yields determined by ${ }^{1} \mathrm{H}$ NMR spectroscopy using dioxane as internal standard. Average of at least three runs. $c$ Moles product per mole catalyst per hour based on total palladium content.

Reasoning that an aqueous phase compatible catalyst could be well-suited to facile separation and recovery, a recycle experiment was conducted by extracting the product and unreacted substrate into ethyl acetate before recharging the aqueous solution of catalyst with a further portion of 
nitrobenzene and re-pressurizing the reactor with hydrogen The data in Figure 5a shows a drop in yield over the first three cycles after which the conversion profile remains stable. ICP analysis of the aqueous phase collected after run five revealed that the palladium content had dropped by $39 \%$ from $42 \mathrm{ppm}$ (0.5 mol\%) to $26 \mathrm{ppm}$ which might account for the gradual decrease in conversion over several recycles. These loses may result from adhesion of insoluble catalyst to the surface of the reactor and glassware during the separation-recovery protocol as ICP analysis on the combined organic extracts confirmed that leaching was negligible $(<0.1 \mathrm{ppm})$. However, attrition was visually most evident during the first three runs after which the reaction mixture appeared homogeneous and thus we cannot discount the possibility that soluble catalyst is responsible for the observed conversions. In this regard, TEM analysis of the solution after the sixth run revealed an increase in the mean particle diameter to $3.05 \pm 0.86 \mathrm{~nm}$ compared with that of $1.93 \pm 0.67 \mathrm{~nm}$ measured for a freshly prepared sample of 2c (Figure 5b,c); this corresponds to a decrease in surface area of $c a$. $35 \%$ which appears to correlate with the drop in conversion.

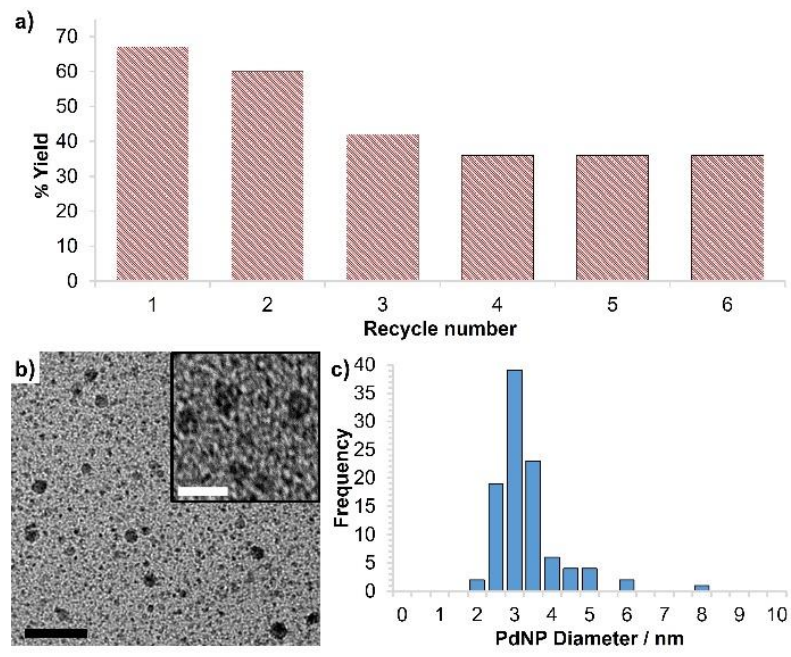

Fig. 5 (a) Recycle study for the hydrogenation of nitrobenzene in water catalysed by PdNP@PPh ${ }_{2}$-PEGPIILP (2c) and (b) HRTEM image of catalyst after 6th run and (c) particle size distribution for $\mathbf{2 c}$ after five recycles showing an average NP size of $3.05 \pm 0.86 \mathrm{~nm}$. Black and white scale bars are $10 \mathrm{~nm}$ and $5 \mathrm{~nm}$, respectively

\section{Transfer Hydrogenation of Nitroarenes}

The encouraging performance of $\mathbf{2} \mathbf{c}$ as a catalyst for the hydrogenation of nitroarenes prompted us to extend our studies to include a comparison of its efficacy as a catalyst for the aqueous phase reduction of nitroaromatic compounds using sodium borohydride as the reducing agent. Relying on recent literature protocols as a lead, a series of catalytic reactions were first conducted with nitrobenzene as the benchmark substrate as this reduction has recently been catalyzed by PdNP-based systems stabilized by a host of supports including PVP, ${ }^{11}$ graphene oxide/poly(imidazole/imidazolium) nanocomposites, ${ }^{33}$ carbon nitride, ${ }^{39}$ PEGylated imidazolium-based phosphinite ionic liquidmodified magnetic silica core shell nanoparticles, ${ }^{26 r}$ carbon nanospheres, ${ }^{10}$ polymethylhydrosiloxane, ${ }^{14} \quad \mathrm{Nb}_{2} \mathrm{O}_{5}$ nanowires, ${ }^{15}$ $\mathrm{Fe}_{3} \mathrm{O}_{4} @$ dextran particles ${ }^{40}$ and tris(triazolyl)-polyethylene glycol. ${ }^{41}$ Batch reactions first explored the effect of time, solvent, substrate: $\mathrm{NaBH}_{4}$ ratio and rate of addition on conversion, full details of which are presented in Table 4. Complete conversion of nitrobenzene and $100 \%$ selectivity for aniline was obtained in water after $2 \mathrm{~h}$ at $25{ }^{\circ} \mathrm{C}$ using a 0.047 mol\% loading of $\mathbf{2 c}$ and a substrate: $\mathrm{NaBH}_{4}$ ratio of 2.5 , whereas markedly lower yields and selectivities were obtained under the same conditions in ethanol, methanol and a 1:1 mixture of alcohol and water (entries 1-5). The low yields and variable selectivities for aniline in these solvents were associated with incomplete reduction to intermediates such $\mathrm{N}$-phenylhydroxylamine and azoxybenzene (vide infra). Conversions increased quite dramatically with increasing temperature (entries 6-9) such that near complete reduction to aniline in $>99 \%$ selectivity could be obtained after only $20 \mathrm{~min}$ at $80^{\circ} \mathrm{C}$. In comparison, reactions conducted at lower temperatures resulted in incomplete reduction and were less selective for aniline; under these conditions the major product of partial reduction was $\mathrm{N}$-phenylhydroxylamine. Variation of the $\mathrm{NaBH}_{4}$ :substrate ratio revealed that conversions and selectivities dropped quite dramatically as the ratio was reduced below 2.5 (entries 10-12), while an increase in this ratio to 5 or 10 improved both conversion and selectivity (entries 14-16) and a further increase to 25 resulted in $86 \%$ conversion to afford aniline in $68 \%$ selectivity with hydroxylamine as the only significant by-product. A control reaction for the reduction of nitrobenzene conducted in water in the absence of $\mathbf{2} \mathbf{c}$ but with 2.5 equivalents of $\mathrm{NaBH}_{4}$ gave no conversion even after $6 \mathrm{~h}$ which confirmed the active role of the catalyst. For comparison, the reduction of nitrobenzene catalyzed by $0.047 \mathrm{~mol} \% \mathrm{Pd} / \mathrm{C}$ under the same conditions reached $90 \%$ conversion but only $63 \%$ selectivity for aniline after $2 \mathrm{~h}$ (entry 17). Finally, reduction of the catalyst loading to $0.00035 \mathrm{~mol} \%$ gave aniline in $96 \%$ yield and $96 \%$ selectivity after $16 \mathrm{~h}$ at $50{ }^{\circ} \mathrm{C}$; this corresponds to a TON of 274,000 and a TOF of $17,125 \mathrm{~h}^{-1}$. Although the poor solubility of the substrate undoubtedly limits this TOF, comparison with related NP-based systems that also operate under mass transfer control $10,14,15,17,19,33,40,41,43$ reveals it to be the most active NP-based system to be reported for the aqueous phase transfer hydrogenation of nitroarenes. While selectivities at low conversion varied quite dramatically due to incomplete reduction, aniline was obtained as the sole product at complete conversion; as such reactions to explore substrate scope were run to full conversion to ensure high selectivity (vide infra). During the preparation of this manuscript, Uberman and Martin disclosed an elegant and comprehensive study on highly efficient PVP-Pd nanoparticles generated by electrochemical methods. ${ }^{11}$ The TOF of $960 \mathrm{~h}^{-1}$ (97\% conversion) reported for the reduction of nitrobenzene required 4.0 equivalents of sodium borohydride and reactions were conducted in water/ethanol whereas the TOF of $1000 \mathrm{~h}^{-1}$ obtained with $2 \mathrm{c}$ only required 2.5 equivalents of $\mathrm{NaBH}_{4}$ and the reaction was conducted in water. A more meaningful comparison was obtained by performing the reduction of nitrobenzene with $\mathbf{2 c}$ under the same conditions described for PVP-PdNP (4.0 equivalents $\mathrm{NaBH}_{4}, 6: 1$ ethanol:water, room temperature, $1 \mathrm{~h})$. Gratifyingly, the TOF of 


\section{Journal Name}

\section{ARTICLE}

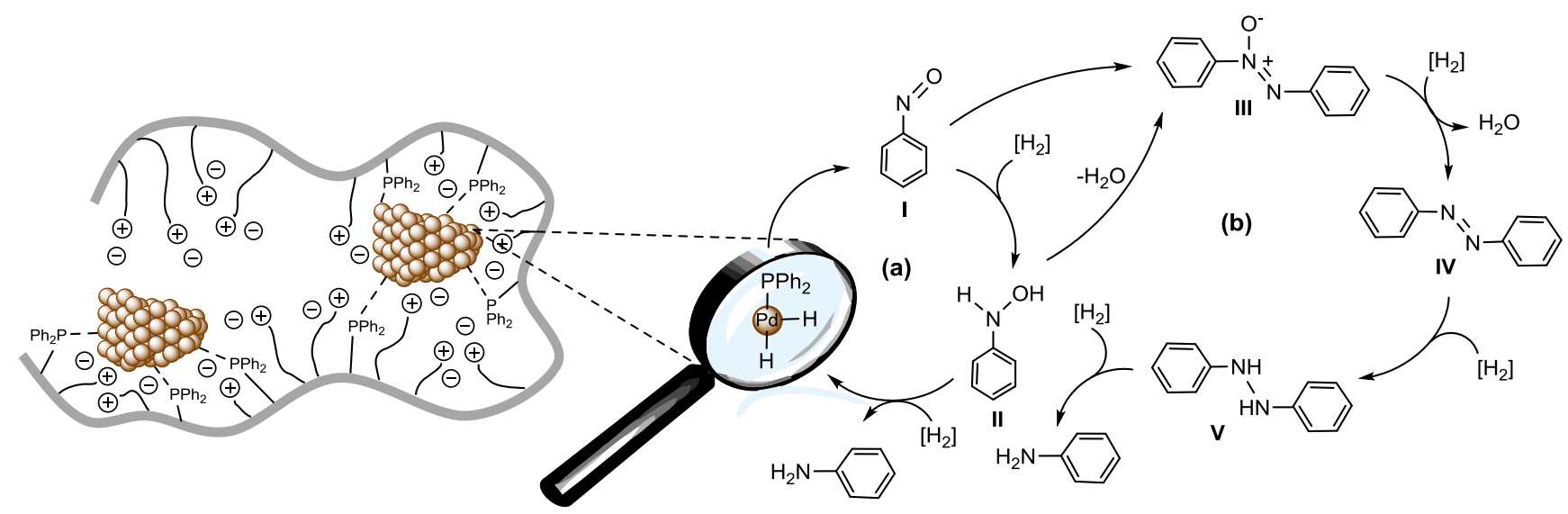

Scheme 1 General mechanisms for the hydrogenation of nitroarenes (a) direct pathway (b) condensation

Table 4 Reduction of nitrobenzene by $\mathrm{NaBH}_{4}$ as a function of catalyst, solvent, temperature and hydrogen source. ${ }^{a}$

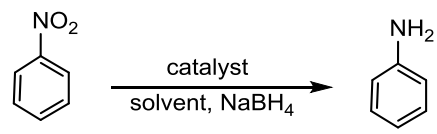

\begin{tabular}{|c|c|c|c|c|c|c|}
\hline Catalyst & Solvent & $\begin{array}{c}\mathrm{NaBH}_{4} \\
\text { equiv }\end{array}$ & $\begin{array}{c}\text { Temp } \\
\left({ }^{\circ} \mathrm{C}\right)\end{array}$ & $\begin{array}{l}\text { Time } \\
(\min )\end{array}$ & $\begin{array}{l}\text { Conv } \\
(\%)^{b}\end{array}$ & $\begin{array}{l}\text { Select } \\
(\%)^{c}\end{array}$ \\
\hline $2 c$ & water & 2.5 & 25 & 120 & 100 & 100 \\
\hline 2c & ethanol & 2.5 & 25 & 120 & 40 & 14 \\
\hline 2c & methanol & 2.5 & 25 & 120 & 33 & 27 \\
\hline $2 c$ & EtOH/water & 2.5 & 25 & 120 & 77 & 17 \\
\hline 2c & $\mathrm{MeOH} /$ water & 2.5 & 25 & 120 & 91 & 27 \\
\hline 2c & water & 2.5 & 20 & 20 & 26 & 38 \\
\hline $2 c$ & water & 2.5 & 40 & 20 & 51 & 65 \\
\hline $2 c$ & water & 2.5 & 60 & 20 & 66 & 73 \\
\hline 2c & water & 2.5 & 80 & 20 & 100 & $>99$ \\
\hline 2c & water & 0.5 & 25 & 120 & 11 & 60 \\
\hline $2 c$ & water & 1.0 & 25 & 120 & 35 & 64 \\
\hline 2c & water & 1.5 & 25 & 120 & 76 & 64 \\
\hline $2 c$ & water & 2.0 & 25 & 120 & 81 & 85 \\
\hline 2c & water & 2.5 & 25 & 20 & 37 & 48 \\
\hline $2 c$ & water & 5.0 & 25 & 20 & 67 & 62 \\
\hline 2c & water & 10.0 & 25 & 20 & 78 & 69 \\
\hline 2c & water & 25.0 & 25 & 20 & 86 & 70 \\
\hline 1c & water & 2.5 & 25 & 120 & $>99$ & $>99$ \\
\hline $3 c$ & water & 2.5 & 25 & 120 & 82 & 82 \\
\hline 4c & water & 2.5 & 25 & 120 & 92 & 92 \\
\hline $\mathrm{Pd} / \mathrm{C}$ & water & 2.5 & 25 & 120 & 63 & 63 \\
\hline \multicolumn{7}{|c|}{ 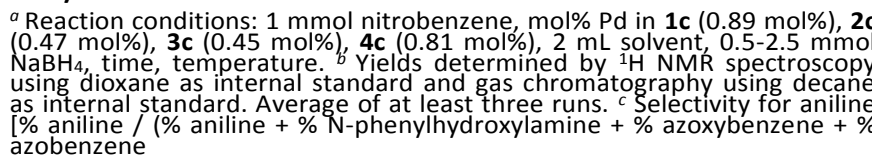 } \\
\hline
\end{tabular}

$1810 \mathrm{~h}^{-1}$ at $85 \%$ conversion is a marked improvement on the $960 \mathrm{~h}^{-1}$ reported for the PVP-PdNPs; however, both systems most likely operate under diffusion control and the TOF of $17,125 \mathrm{~h}^{-1}$ obtained at $50{ }^{\circ} \mathrm{C}$ (vide supra) is probably more representative of the potential activity of $\mathbf{2 c}$.

Two mechanisms have been proposed for the hydrogenation of nitroarenes, ${ }^{1 d, 42}$ the first involves direct hydrogenation via nitroso and hydroxylamine intermediates I and II, respectively, while the second is a condensation route involving azoxy and azo intermediates III and IV, respectively (Scheme 1). While palladium-based catalysts generally operate via direct hydrogenation, ${ }^{1 d, 37,42 a}$ Uberman et al. have recently provided convincing evidence that electrochemically generated PdNPs stabilized by PVP catalyse the hydrogenation of 4-chloronitrobenzene via the condensation pathway; ${ }^{11}$ moreover, high selectivity for the azoxy intermediate could be obtained at low palladium loading and low $\mathrm{NaBH}_{4}$ addition. Interested in exploring the reaction profile for $\mathbf{2 c}$, the reduction of nitrobenzene was monitored and intermediates identified and quantified using ${ }^{1} \mathrm{H}$ NMR spectroscopy and GC, the results of which are presented graphically in Figure 6a-b. Under the standard conditions described above, Nphenylhydroxylamine (II) was identified as the major intermediate (43\%) and only trace quantities of azoxy (III) (4\%) and azo intermediates (IV) (3\%) were detected i.e. $86 \%$ selective for hydroxylamine (Figure 6a). This composition-time profile is consistent with reaction via the direct pathway in contrast to the PdNP-PVP system described above in which azoxy III was identified as the major intermediate. ${ }^{11}$ While it is difficult to provide a definitive explanation for this difference, the surface of the PdNP may well be heavily populated with $\mathrm{PPh}_{2}$ and such crowding could disfavour condensation of two 
molecules of chemisorbed Ar-N-O-H. In comparison, the reaction was markedly slower in the presence of a reduced amount of sodium borohydride (1.0 equivalent) and in this case a significant amount of azoxybenzene (up to $21 \%$ ) was present suggesting that condensation is dominant under these conditions (Figure 6b). In this regard, we note that low concentrations of hydrogen have previously been reported to favour the condensation pathway. ${ }^{43}$ Thus, it may well ultimately be possible to identify an optimum catalyst and conditions to achieve high yields and selectivities for commercially important azoxy-based compounds.
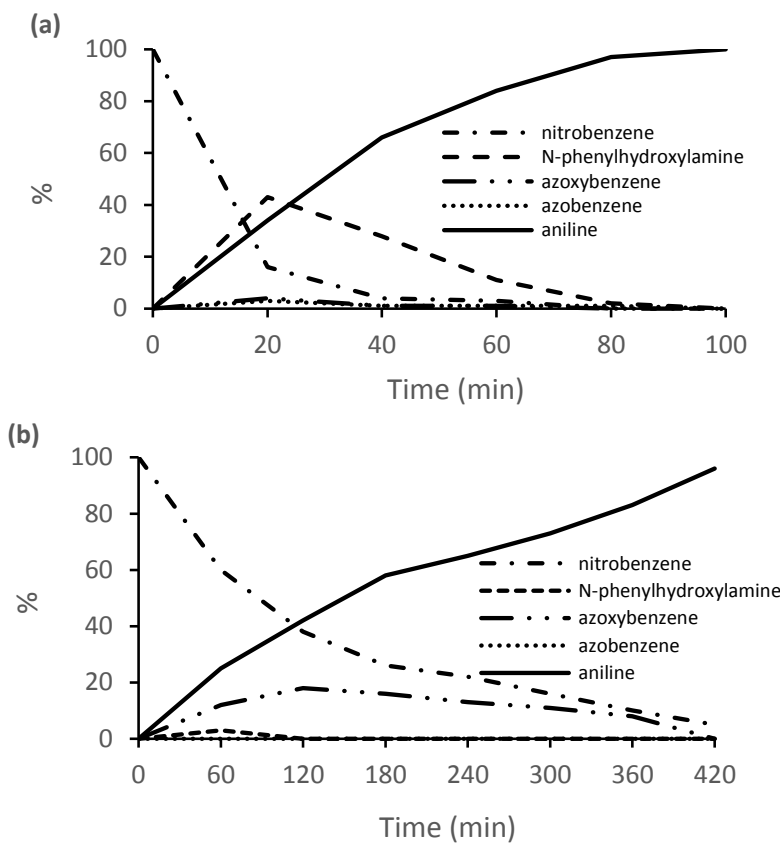

Fig. 6 (a) Reaction profile as a function of time for the reduction of nitrobenzene in water at $25^{\circ} \mathrm{C}$ using 2.5 mole equivalents of $\mathrm{NaBH}_{4}$ and a 0.05 mol\% loading of 2c, showing direct hydrogenation via $\mathrm{N}$-phenylhydroxylamine to be the major pathway, (b) reaction composition as a function of time for the reduction of nitrobenzene in water using 1 mole equivalent of $\mathrm{NaBH}_{4}$ and a 0.05 mol\% loading of catalyst.

A series of kinetic studies conducted on the hydrogenation of 4-nitrophenol were used to quantify and compare the performance of catalysts $\mathbf{1 c} \mathbf{c} \mathbf{- 4}$. This transformation is typically chosen to obtain rate data because the reaction can be monitored by UV-vis spectroscopy as the 4-nitrophenolate anion has a characteristic absorption band at $400 \mathrm{~nm}$ which decreases with time and the 4-aminophenolate product has a distinctive absorption band at $300 \mathrm{~nm}$. The associated plots of $\ln \left(C_{t} / C_{0}\right)$ against time for the reduction of 4-nitrophenolate catalyzed by $\mathbf{1 c}-\mathbf{4 c}$ appear to be linear which is consistent with related studies that report pseudo first order kinetics for this reduction (Figure 7); 44 even though this data appears to be a reasonable fit deviations from linearity may well be attributed to mass transfer effects. The associated rate constants together with the corresponding activity parameters $\mathrm{k}$, the ratio between the rate constant and the moles of catalyst, are summarized in Table 5 . The activity parameter allows a more meaningful comparison to be undertaken as it takes the catalyst loading into account and since the reaction volume is constant it provides a more realistic measure of the performance. ${ }^{40}$ To this end, the data in Table 5 confirms that 2c has the highest activity parameter, while that for $\mathbf{3 c}$ is marginally lower which is consistent with the conversions obtained in the optimization studies described above (Table 4).

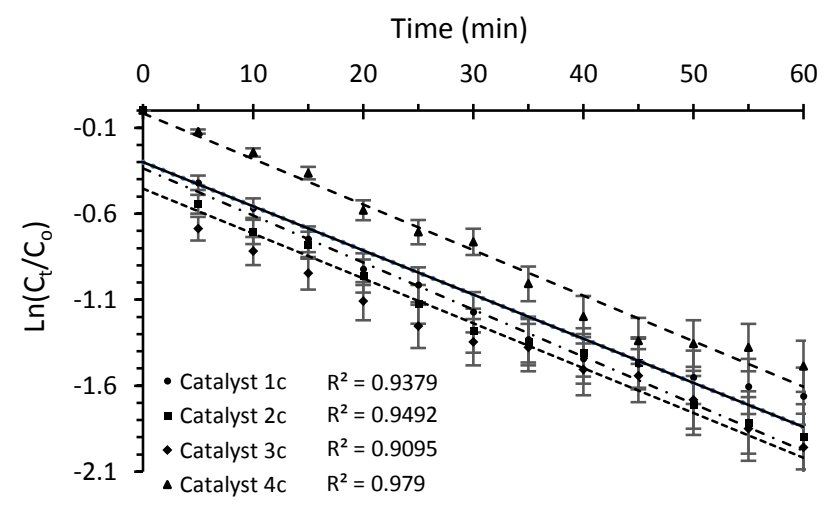

Fig. 7 Plots of $\ln \left(\mathrm{C}_{\mathrm{t}} / \mathrm{Co}\right)$ versus time for the reduction of 4-nitrophenol $(5.0 \mathrm{mmol})$ using 2.5 equivalents of $\mathrm{NaBH}_{4}$ catalysed by $\mathbf{1 c}(0.089 \mathrm{~mol} \%), 2 \mathrm{c}(0.047 \mathrm{~mol} \%), 3 \mathrm{c}(0.045$
$\mathrm{mol} \%), 4 \mathrm{c}\left(0.081 \mathrm{~mol}^{2}\right)$.

Table 5 Summary of $k_{\mathrm{obs}}$ and activity parameters (к) for catalysts 1c-4c

\begin{tabular}{lcccc}
\hline Parameter/catalyst & 1c & 2c & 3c & 4c \\
\hline$k_{\text {obs }}\left(\mathrm{min}^{-1}\right)$ & 0.025 & 0.027 & 0.025 & 0.026 \\
$\mathrm{k}\left(\mathrm{min}^{-1} \mathrm{~mol}^{-1}\right)$ & 5,620 & 11,500 & 11,110 & 6,420 \\
\hline
\end{tabular}

The protocol developed above has also been applied to the aqueous phase reduction of a selection of nitroarenes to compare the performance of pre-reduced PdNP@PPh ${ }_{2}$-PEGPIILP (2c) against catalyst generated from its precursor $\mathrm{PdCl}_{4} @ \mathrm{PPh}_{2}$-PEGPIILP (1c) as well as to explore the scope of substrates and evaluate the efficiency against existing systems; all reactions were run to high conversion in order to obtain the corresponding amine as the sole product. The data in Table 6 shows that 2c efficiently reduces nitroarenes containing electron-donating and electronwithdrawing groups to afford the corresponding aniline as the sole product in high yield at room temperature after relatively short reaction times; typically 1-6 $\mathrm{h}$ (entries 2-6). The high selectivity for the aniline over other intermediate reduction products such as azoxyarenes, hydroxylamines, oximes and nitrones enabled a straightforward isolation and purification protocol to be developed. Nitroarenes substituted at the 2-position were also converted to the corresponding aniline in high yield and $100 \%$ selectivity, albeit after longer reaction times due to steric hindrance inhibiting access of the substrate to the catalyst surface. Similarly, reduction of 1-nitronaphthalene was sluggish under the same conditions with a yield of $79 \%$ after $6 \mathrm{~h}$, however, complete conversion to 1-aminonaphthalene could be obtained in the same time when the reaction temperature was raised to $50{ }^{\circ} \mathrm{C}$. Hydrogenation of 1-chloro-4-nitrobenzene occurred with high selectivity to afford 4-chloroaniline in $93 \%$ yield together with a 
minor amount of aniline (6\%) resulting from hydrodechlorination. In contrast, hydrodechlorination of 2-chloro-1-nitrobenzene was markedly more facile under the same conditions such that a 39:61 mixture of 2-chloroaniline and aniline was obtained after $6 \mathrm{~h}$. Unfortunately, 2c was unable to reduce heteroaromatic nitro compounds such as 2- and 3-nitropyridine or 6- and 8nitroquinoline as quantitative amounts of starting material were consistently recovered even after an extended reaction time of 4 h. This is perhaps not too surprising as there are only a handful of reports of the reduction of nitro-substituted heteroaromatics catalysed by PdNP-based systems; notably these typically use alternative reducing agents such as ethanol or polymethylhydrosiloxane and require reaction temperatures as high as $80^{\circ} \mathrm{C}$ as well as high catalyst loadings and/or long reaction times. ${ }^{9 b, 14}$ One exception is a ligand free $\mathrm{Fe} / \mathrm{ppm} \mathrm{Pd}$ nanoparticles system stabilized by the PEG containing designer surfactant TPGS750-M developed by Lipshutz which catalyses the reduction of several nitro containing heterocycles to give good yields of amine. ${ }^{22}$ In an attempt to investigate the extent to which pyridinebased substrates may poison/inhibit the catalyst or modify the course of the reaction the reduction of nitrobenzene was monitored as a function of time after pre-treating the catalyst with one equivalent of pyridine for 10 minutes immediately prior to addition of the substrate. The resulting composition-time profile in Figure 8 reveals a marked difference compared with that in the absence of pyridine (Figure 6a) as significant quantities of azoxy and azo intermediates were identified together with $\mathrm{N}$ phenylhydroxylamine i.e. in the presence of pyridine condensation appears to compete with direct hydrogenation. However, further studies will be required to understand why $\mathbf{2 c}$ does not catalyse the reduction of $\mathrm{N}$-heterocycles. To this end, a catalytic reaction mixture containing 2-nitrotoluene and 5-nitroindole gave $4 \%$ conversion to 2-nitrotoluene after $1 \mathrm{~h}$ whereas the corresponding reaction conducted in the absence of 5 -nitroindole gave $46 \%$ conversion in the same time. Moreover, addition of 5-nitroindole to a sodium borohydride mediated reduction of 2-nitrotoluene after a reaction time of $1 \mathrm{~h}$ resulted in immediate deactivation as the conversion remained at $46 \%$ even after the reaction time was extended to $2 \mathrm{~h}$.

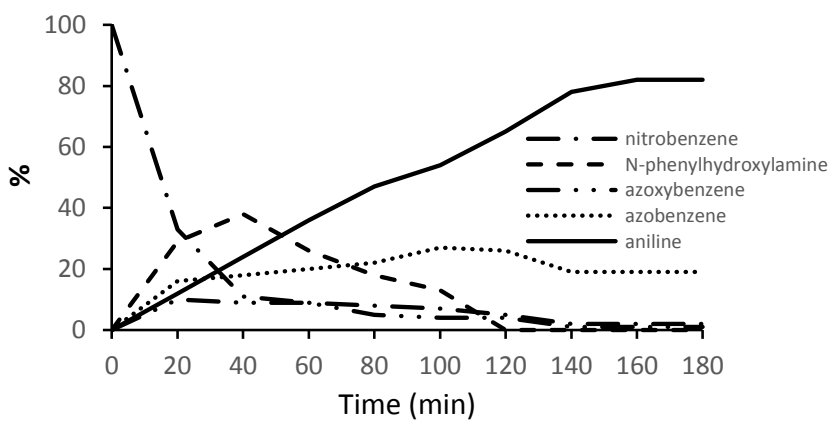

Fig. 8 Reaction composition as a function of time for the reduction of nitrobenzene $(1.0 \mathrm{mmol})$ in water at $25^{\circ} \mathrm{C}$ using 2.5 mole equivalents of $\mathrm{NaBH}_{4}$, and $0.05 \mathrm{~mol} \% \mathbf{2 c}$ in the presence of one equivalent $(1.0 \mathrm{mmol})$ of pyridine showing that the condensation pathway competes with direct reduction via $N$ phenylhydroxylamine.
Table 6 Reduction of nitroarenes by $\mathrm{NaBH}_{4}$ using $\mathrm{PdCl}_{4} @ \mathrm{PPh}_{2}$-PEGPIILP (2b) or PdNP@PPh ${ }_{2}$-PEGPIILP (2c) ${ }^{a}$

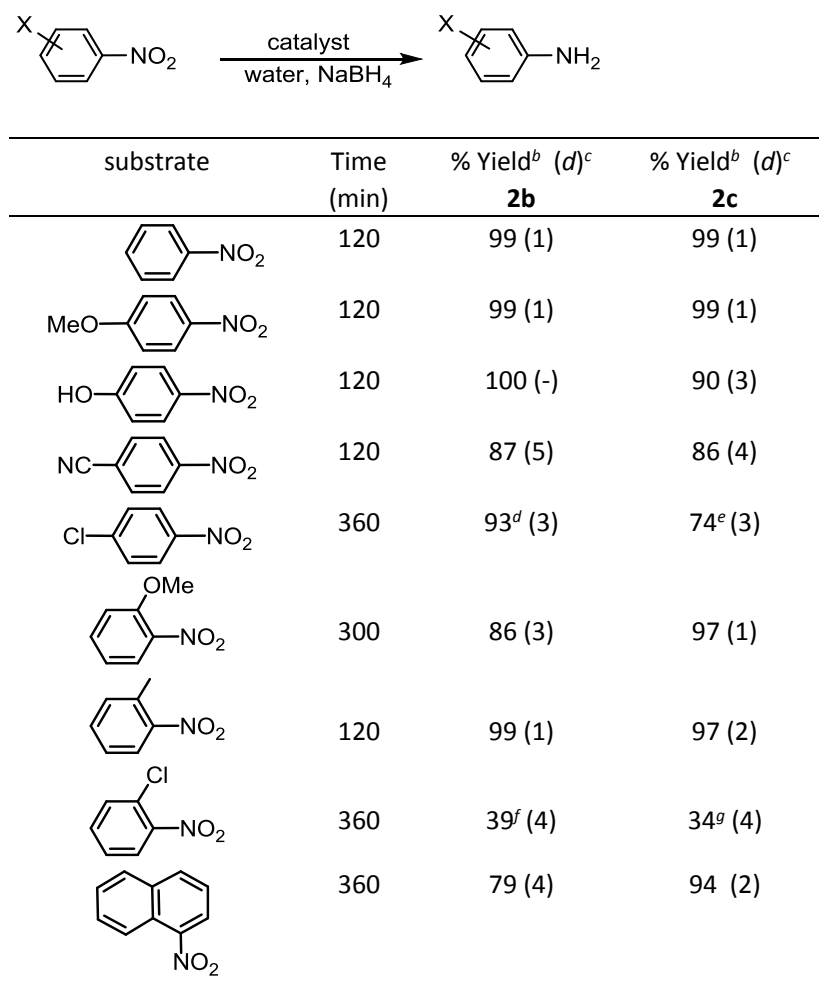

${ }^{a}$ Reaction conditions: $1 \mathrm{mmol}$ nitrobenzene, $0.05 \mathrm{~mol} \% \mathbf{2 b}$ or $\mathbf{2 c}, 10 \mathrm{~mL}$ water, $2.5 \mathrm{mmol} \mathrm{NaBH}_{4}, 25{ }^{\circ} \mathrm{C}$, time. ${ }^{b}$ Yields determined by ${ }^{1} \mathrm{H}$ NMR spectroscopy using dioxane as internal standard and gas chromatography using decane as internal standard. Average of at least three runs. ${ }^{c}$ average deviation $(d)=\left(\left|\mathrm{x}_{1}-\bar{x}\right|+\mid \mathrm{x}_{2}\right.$ $\left.-\bar{x}|+| \mathrm{x}_{3}+\bar{x} \mid\right) / 3$ where $\bar{x}=\left(\mathrm{x}_{1}+\mathrm{x}_{2}+\mathrm{x}_{3}\right) / 3$ and $\mathrm{x}_{\mathrm{n}}$ is the conversion for run $\mathrm{n}^{d}$ $6 \%$ aniline formed. $e 7 \%$ aniline formed. ${ }^{f} 61 \%$ aniline formed. $g 66 \%$ aniline formed.

As $\mathbf{2 c}$ is generated in an aqueous phase sodium borohydride reduction of $\mathrm{PdCl}_{4} @ \mathrm{PPh}_{2}$-PEGPIILP (2b), we became interested in exploring whether this precursor could be used to generate catalyst in situ immediately prior to addition of the substrate. Thus, a series of comparative reactions were conducted using a 0.05 mol\% loading of $\mathbf{2} \mathbf{b}$ and, gratifyingly, for each substrate tested yields were comparable to those obtained with $\mathbf{2 c}$, for the same reaction time (Table 6 ). This protocol could be potentially advantageous as the precatalyst has a long shelf-life and it would also eliminate the need to prepare, isolate and store nanoparticle catalysts. TEM analysis of in situ generated $\mathbf{2 c}$ and $\mathbf{4 c}$ revealed that the resultant nanoparticles are monodisperse with average particle diameters of $3.36 \pm 0.61 \mathrm{~nm}$ and $2.55 \pm 0.97 \mathrm{~nm}$, respectively, compared with $1.93 \pm 0.96 \mathrm{~nm}$ and $4.02 \pm 1.11 \mathrm{~nm}$ for their pre-reduced counterparts (Figure 9a-d). Interestingly, this change of ca. $40 \%$ for both does not appear to be reflected in the conversions as $\mathbf{2 b}$ and $\mathbf{2 c}$ both gave 99\% conversion for the transfer hydrogenation of nitrobenzene while $\mathbf{4 b}$ and $\mathbf{4 c}$ gave conversion of $93 \%$ and $92 \%$, respectively, i.e. surface modification by the phosphine may well dominate activity rather than particle size, although further studies will be required to substantiate the extent to which Pd----P interactions modify the electronic 
properties and catalyst efficacy. However, at this stage we cannot exclude the possibility that the observed conversions are dispersion controlled, particularly based on a comparison of the TOFs obtained with $\mathbf{2 c}$ and $\mathbf{5 c}$ as the NPs in both systems have similar mean diameters (vide infra).
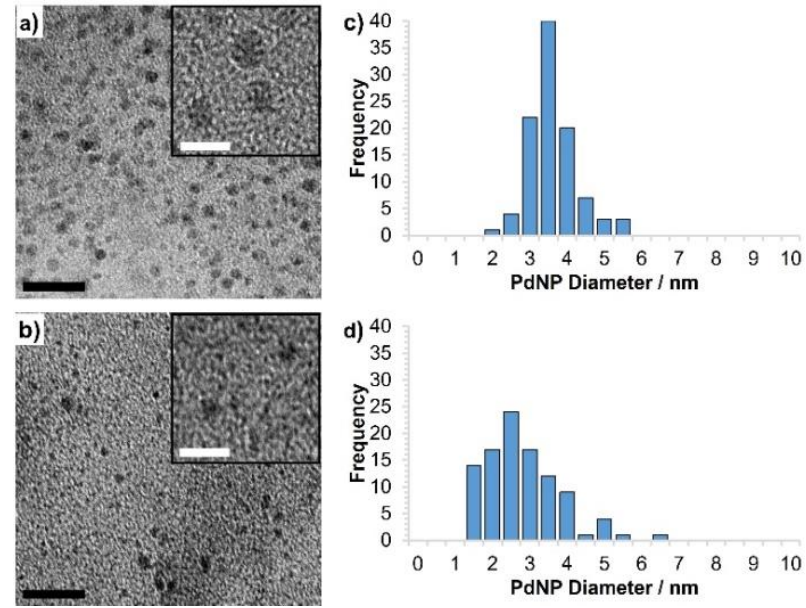

Figure 9. HRTEM images of in situ generated (a) PdNP@PPh ${ }_{2}-$ PEGPIILP (2c) and (b) PdNP@PSty ${ }_{3}$-PEGPIILP (4c) and (c, and d) corresponding particle size diameters are $3.36+0.61 \mathrm{~nm}$ and $255+0.97 \mathrm{~nm}$ for $\mathbf{2 c}$ and $4 \mathrm{c}$, respectively. Black and white scale bars are 5 and $10 \mathrm{~nm}$, respectively.

While the TOF's obtained under transfer hydrogenation with $\mathbf{2 c}$ are markedly higher than those for hydrogenation, the former protocol is not tolerant towards reducible groups such as ketones and esters or heteroaromatic compounds which appear to deactivate the catalyst. However, $\mathbf{2 c}$ is an efficient catalyst for both protocols under mild operating conditions which will provide a platform for further studies to develop improved systems.

The efficacy of $\mathbf{2 c}$ for the hydrogenation of nitroarenes under mild conditions prompted us to explore the potential for use in a continuous flow process as this should overcome the problem of catalyst loss, allow straightforward product separation and, moreover, be amenable to scale-up. Three alterations to the reaction conditions were required to successfully transfer the process to continuous flow: (i) a 1:1 solvent mixture of ethanol and water was used to solubilise reactants/products; (ii) $\mathrm{NaBH}_{4}$ was dissolved in $1 \mathrm{M} \mathrm{NaOH}_{(\mathrm{aq})}$ $(\mathrm{pH} \mathrm{14})$ to reduce the rate of hydrolysis (half-life $=426$ days compared to 3.7 seconds at $\mathrm{pH} 7$ ); ${ }^{45}$ (iii) the reaction temperature was increased to $60{ }^{\circ} \mathrm{C}$ to compensate for the reduced rate of reduction observed when using an ethanolwater solvent mixture. The packed-bed reactor consisted of a $2.5 \mathrm{~mL}$ aluminium tube packed with catalyst $2 \mathrm{c}(92 \mathrm{mg}, 3.81$ wt\% Pd) and sand. A schematic of the set-up is shown in Scheme 2 and further details are provided in the ESI. In summary, a $99 \%$ yield of the desired aniline product was achieved under these conditions, with no sign of catalyst deactivation after a period of $250 \mathrm{~min}$ (20 reactor volumes). The space-time yield (STY) of the process was almost twice that of its batch counterpart (0.738 cf. $\left.0.384 \mathrm{~g} \mathrm{~L}^{-1} \mathrm{~min}^{-1}\right)$.
Following these preliminary results, work is currently underway to develop an automated system for the optimisation of reaction conditions for productivity and green metrics.

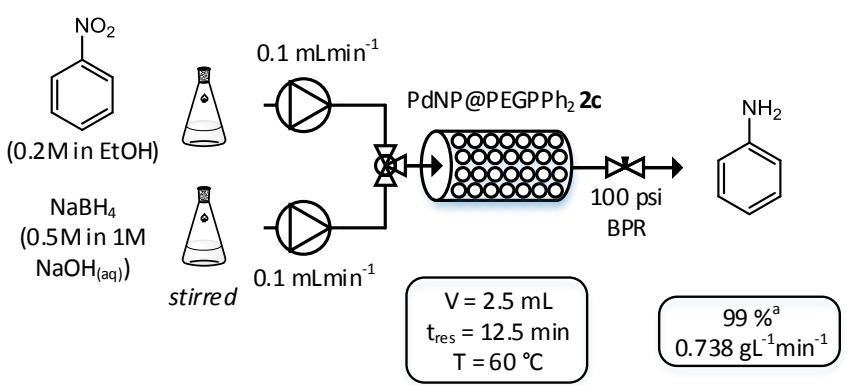

Scheme 2. Flow set-up for the transfer hydrogenation of nitrobenzene using sodium borohydride and a PdNP@PPh ${ }_{2}$-PEGPIILP (2c) packed bed reactor.a Aniline yield determined by HPLC analysis using 1,3,5-trimethoxybenzene as internal standard.

The efficiency of $\mathbf{2 c}$ as a hydrogenation catalyst prompted us to explore its potential to catalyse an aqueous phase tandem Suzuki-Miyaura cross coupling-hydrogenation sequence as a practical single-pot protocol for the synthesis of biaryl amines (Scheme 3). In order for this tandem sequence to be realized the conditions for each individual reaction must be compatible. Fortunately, to this end, a preliminary control Suzuki-Miyaura cross-coupling between 3- or 4-bromobenzene and phenylboronic acid gave a near quantitative yield of product after only $3 \mathrm{~h}$ under these optimum conditions. After this time, an aqueous solution of $\mathrm{NaBH}_{4}$ (2.5 equiv.) was added and the resulting mixture maintained at room temperature for a further $8 \mathrm{~h}$. Gratifyingly, the two substrates examined 3- and 4-bromo-1-nitrobenzene gave the 3- and 4-aminobiphenyl, respectively, in excellent isolated yield. These are the first examples of single-pot tandem SuzukiMiyaura coupling-hydrogenation reaction sequences conducted at room temperature in aqueous media and future studies will explore the range of aryl boronic acids as well as complimentary reaction combinations between nitrobenzene boronic acids and aryl/heteroaryl bromides. There are two reports of this type of consecutive reaction but in each case the reaction was conducted in ethanol or an ethanol/water mixture and either a markedly higher catalyst loading and/or elevated reaction temperature was required; however, good conversions were obtained in both cases. ${ }^{11,46}$

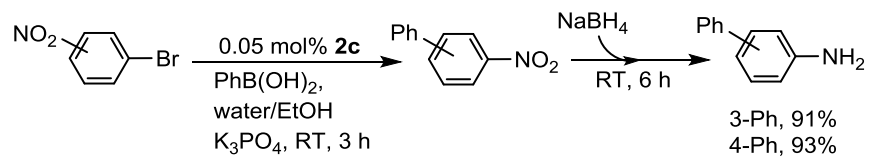

Scheme 3. Single-pot Suzuki-Miyaura cross coupling hydrogenation sequence for the synthesis of biarylamines catalysed by $\mathbf{2 c}$. 


\section{Conclusions}

This study has demonstrated that PEG-modified phosphinedecorated polymer immobilised ionic liquid-stabilised palladium nanoparticles are highly efficient and chemoselective catalysts for the aqueous phase hydrogenation of a range of aromatic and heteroaromatic nitroarenes at low catalyst loadings under mild conditions. While the same systems also catalyse the aqueous phase sodium borohydridemediated reduction of nitroaromatics with remarkable efficacy, they do not catalyse the transfer hydrogenation of heteroaromatic nitroarenes or nitroarenes with reducible groups such as ketones or esters. Moreover, even though reactions have been shown to occur under diffusion control, PdNP@PPh ${ }_{2}$-PIILP is the most efficient NP-based system to be reported for the aqueous phase hydrogenation and transfer hydrogenation of nitroarenes under mild conditions. Differences in catalyst efficacy arising from selective modification of the support have been attributed to changes in the balance of hydrophilicity/hydrophobicity which affects catalyst dispersibility and facilitates access to the active site. Although it is not possible to fully deconvolute how each component influences catalyst performance, for instance, dispersibility, solubility, NP size and surface modification, this study has shown that each of the ionic liquid, PEG and phosphine components is necessary to achieve optimum efficiency. Interestingly, larger nanoparticles formed when the heteroatom donor was either isolated in the cross-linker or completely absent which may well indicate that the heteroatom donor influences nucleation and NP growth: however, further studies will be required to firmly establish the role of the heteroatom donor in nanoparticle formation and whether it influences/modifies surface electronic structure and reactivity. To this end, during the review of this manuscript, Yang reported that platinum nanoparticles stabilised by triphenylphosphine modified silica are highly chemoselective catalysts for the hydrogenation of acetophenone and phenylacetylene; the efficacy of this system was proposed to arise from a strong interaction between the phosphine donor and platinum NPs. ${ }^{47}$ While batch recycle studies suffered from catalyst loss during the recovery procedure, preliminary studies using a packed-bed reactor under continuous flow operation were highly encouraging as good yields and a stable catalyst profile were achieved over $4 \mathrm{~h}$ and, gratifyingly, the corresponding space-time-yield was twice that obtained in batch. The optimum system has been used to develop a tandem Suzuki-Miyaura cross coupling nitro reduction sequence and the corresponding biaryl amines were obtained in excellent yields from an operationally straightforward single-pot procedure. In summary, PEG-based phosphine-modified polymer immobilized ionic liquidstabilized PdNPs catalyse the reduction of a range of aromatic and heteroaromatic nitro compounds under extremely mild operating condition; moreover, catalyst generated in situ either competes with or outperforms its preformed counterpart which offers a number of practical benefits. The concept of heteroatom donor-modified PIIL-stabilised NPs will provide a strong platform to develop alternative PIILP-based metal nanoparticles and bimetallic systems to diversify the range of catalytic transformations and applications. The current emphasis/challenge is to identify and develop a system that will enable catalyst rates to be determined.

\section{Conflicts of interest}

There are no conflicts to declare.

\section{Acknowledgements}

We gratefully acknowledge Newcastle University for financial support (TB). Solid state ${ }^{31} \mathrm{P}$ and ${ }^{13} \mathrm{C} N M R$ spectra were obtained at the EPSRC UK National Solid State NMR Service at Durham University, high resolution mass spectra were obtained at the ESPRC National Mass Spectrometry Service in Swansea, XPS data was obtained at NEXUS the National EPSRC XPS Users Service at Newcastle University. FD-XAS was performed at BM28 at the ESRF (Experiment no. $\mathrm{CH}-5045$ ) with help from Dr Laurence Bouchenoire and Dr Paul Thompson. We also thank $\mathrm{Dr}$ Maggie White and Dr Rantej Kler for collecting the powder X-ray diffraction data (Newcastle) and Dr Kathryn White for the SEM images (Faculty of Medical Sciences, Newcastle University). The authors also sincerely thank reviewer 2 for providing constructive, insightful, welldirected and highly informative feedback and comments.

\section{Notes and references}

1 (a) N. Ono, The Nitro Group in Organic Synthesis, Wiley-VCH, New York, 2001; (b) H.-U Blaser and E. Schmidt, Heterogeneous Catalysis and Fine Chemicals, Vol 4, Elsevier, Amsterdam, 1997; (c) Z. Rappoport, The Chemistry of Anilines, Wiley, 3007; (d) H.-U. Blaser, S. Steiner and M. Struder, ChemCatChem, 2009, 1, 210-221; (e) H.U. Blaser, Science, 313, 312-313; (f) H. K. Kadam and S. G. Tilve, RSC Adv., 2015, 5, 83391-83407; (g) R. S. Downing, P. J. Kunkeler and H. Bekkum, Catal. Today, 1997, 37, 121-136.

2 (a) D. S. Surry and S. L. Buchwald, Angew. Chem. Int. Ed., 2008, 47, 6338-6361; (b) C. Thomas, M. Wu and K. Billingsley, J. Org. Chem., 2016, 81, 330-335; (c) Y. Aubin, C. Fischmeister, C. M. Thomas, and J. L. Renaud, Chem. Soc. Rev., 2010, 39, 4130-4145; (d) J. F. Hartwig, Acc. Chem. Res., 2008, 41, 1534-1544.

3 (a) S. Semwal and J. Choudhury, ACS Catal., 2016, 6, 24242428; (b) T. C. Nugent and M. El-Shazly, Adv. Synth. Catal., 2010, 352, 753-819; (c) S. Elangovan, C. Topf, S. Fischwer, H. Jiao, A. Spannenberg, W. Baumann, R. Ludwig, K. Junge and M. Beller, J. Am. Chem. Soc., 2016, 138, 8809-8814.

4 (a) S. M. Kelly and B. H. Lipshutz, Org. Lett., 2014, 16, 98-101; (b) Z. Zhao, H. Yang, Y. Li and X. Guo, Green Chem., 2014, 16, 1274-1281; (c) F. Zamani and S. Kianpour, Catal. Commun., 2014, 45, 1-6; (d) P. S. Rathore, R. Patidar, T. Shripathi and S. Thakore, Catal. Sci. Technol., 2015, 5, 286-295; (e) R. J. Kalbasi, F. Zamani, RSC Adv., 2014, 4, 7444-7453; (f) M. M. Moghaddam, B. Pieber, T. Glasnov and C. O. Kappe, ChemSusChem, 2014, 7, 3122-3131.

5 (a) P. Wang, H. Liu, J. Niu, R. Li and J. Ma, Catal. Sci. Technol., 2014, 4, 1333-1339. (b) Y. M. Yamada, Y. Yuyama, T. Sato, S. Fujikawa and Y. Uozumi, Angew. Chem Int. Ed., 2014, 53, 
127-131. (c) X. Liu, H.-Q. Li, S. Ye, Y. M. Liu, H. Y. He and Y. Cao, Angew. Chem. Int. Ed., 2014, 53, 7624-7628. (d) S. G. Oh, V. Mishra, J. K. Cho, B.-J. Kim, H. S. Kim, Y.-W. Suh, H. Lee, H. S. Park and Y. J. Kim, Catal. Commun., 2014, 43, 7983.

6 (a) V. Macho, L. Vojcek, M. Schmidtova, and M. Harustiak, J. Mol. Cat., 1994, 88, 177-184; (b) U Sharma, P. Kumar, N, Kumar, V. Kumar and b. Singh, Adv. Synth. Catal., 2010, 352 1834-1840; (c) Q. Shi, R. Lu, K. Jin, Z. Zhang and D. Zhao, Green Chem., 2006, 8, 868-870; (d) D. Cantillo, M. M. Moghaddam, and O. C. Kappe, J. Org. Chem., 2013, 78, 45304542; (e) L. Huang, P. Luo, W. Pei, X. Liu, Y. Wang, J. Wang W. Xing and J. Huang, Adv. Synth. Catal., 2012, 354, 26892694.

7 G. Rothenberg, Catalysis, Concepts and Green Applications, Wiley-VCH, Weinheim, 2008.

8 (a) L. Vaccaro, D. Lanari, A. Marrocchi and G. Strappaveccia, Green Chem., 2014, 6, 3680-3704; (b) T. Noel and S. L. Buchwald, Chem. Soc. Rev., 2011, 40, 5010-5029. (c) V. Heeesel, D. Kralisch, N. Kockmann, T. Noel and Q. Wang, ChemSusChem, 2013, 6, 746-789.

9 (a) O. Verho, K. P. J. Gustafson, A. Nagendiran, C.-W. Tai and J.-E. Bäckvall, ChemCatChem, 2014, 6, 3153-3159; (b) O. Verho, K A. Nagendiran, C.-W. Tai, E. V. Johnston and J.-E. Bäckvall, ChemCatChem, 2014, 6, 205-211.

10 Y.-M. Lu, H.-Z. Zhu, W.-G. Li, B. Hu and S-H. Yu, J. Mat. Chem. A, 2013, 1, 3783-3788.

11 P. M. Uberman, C. S. Garcia, J. R. Rodriguez and S. E. Martin, Green Chem., 2017, 19, 739-748.

12 D. R. Petkar, B. S. Kadu and R. C. Chikate, RSC Adv., 2014, 4, 8004-8010.

13 Y. Su, X. Li, Y. Wang, H. Zhong and R. Wang, Dalton Trans., 2016, 45, 16896-16903.

14 D. Damodara, R. Arundhathi, T. V. R. Babu, M. L. Legan, H. J. Kumpaty and P. R. Likhar, RSC Adv., 2014, 4, 22567-22574.

15 Z. Zhang, F. Wang, C. Chen, T. Zhang, X. Jiang and Z. Yun, RSC Adv., 2014, 4, 45088-45094.

16 P. Wang, H. Zhu, M. Liu, J. Niu, B. Yuan, R. Li and J. Ma, RSC Adv., 2014, 4, 28922-28927.

17 F. Yang, A. Feng, C. Wang, S. Dong, C. Chi, X. Ja, L. Zhang and Y. Li, RSC Adv., 2016, 6, 16911-16916.

18 Z. Wu and H. Jiang, RSC Adv., 2015, 5, 34622-34629.

19 E. Kim, H. S. Jeong and B. M. Kim, Catal. Commun., 2014, 45, 25-29.

20 Z. Li, J. Li, J. Liu, Z. Zhao, C. Xia and F. Li, ChemCatChem, 2014, 6, 1333-1339.

21 F. Yang, C. Wang, L. Wang, C. Liu, A. Feng, X. Liu, C. Chi, X. Jia, L. Zhang and Y. Li, RSC Adv., 2015, 5, 37710-37715.

22 J. Feng, S. Handa, F. Gallou and B. H. Lipshutz, Angew. Chem. Int. Ed., 2016, 55, 8979-8983.

23 (a) J. Dupont, Acc. Chem. Res., 2011, 44, 1223-1231; (b) P. Wasserscheid and T. Welton, Ionic Liquids in Synthesis, Wiley-VCH, Weinheim, 2007; (c) S. Doherty in Catalysis in Ionic Liquids: From Catalyst Synthesis to Applications, Eds. C. Hardacre, V. Parvulescu, RSC Catalysis Series, The Royal Society of Chemistry, 2014, pp 44-308.

24 (a) G. S. Fonseca, A. P. Umpierre, P. F. P. Fichtner, S. R. Teixeira and J. Dupont, Chem.-Eur. J., 2003, 9, 3263-3269. (b) F. Bellina and C. Chiappe, Molecules, 2010, 15, 2211-2245.

25 K. L. Luska and A. Moores, ChemCatChem. 2012, 4, 15341546.

26 For examples of nanoparticles stabilized by heteroatom donor modified ionic liquids, amines (a) A. Zhang and H. Cui, Langmuir, 2009, 25, 2604-2612; (b) Z. Wang, Q. Zhang, D. Kuehner, A. Ivaska and L. Niu, Green Chem., 2008, 10, 907 909. Nitriles: (c) D. B. Zhao, Z. F. Fei, T. J. Geldbach, R. Scopelliti, and P. J. Dyson, J. Am. Chem. Soc., 2004, 126 15876-15882; (d) C. Chiappe, D. Pieraccini, D. Zhao and Z. Fei and P. J. Dyson, Adv. Synth. Catal., 2006, 348, 68-74; (e) Z. Fei, D. Zhao, D. Pieraccini, W. H. Ang, T. J. Geldbach, R. Scopelliti, C. Chiappe and P. J. Dyson, Organometallics, 2007, 26, 1588-1598; (f) X. Yang, Z. Fei, D. Zhao, W. H. Ang, Y. Li and P. J. Dyson, Inorg. Chem., 2008, 47, 3292-3297; (g) M. H. G. Prechtl, J. D.; Scholten and J. Dupont, J. Mol. Cat. A: Chem., 2009, 313, 74-78. Thiolate: (h) H. Itoh, K. Maka and Y. Chujo, J. Am. Chem. Soc., 2004, 126, 3026-3027; (i) N. Kocharova, J. Leiro, J. Lukkari, M. Heinonen, T. Skala, F. Sutara, M. Skoda and M. Vondracek, Langmuir, 2008, 24 3235-3242. Bipyridine: (j) B. Léger, A. Denicourt-Nowicki, A. Roucoux and H. Olivier-Bourbigou, Adv. Synth. Catal., 2008, 350, 153-159; (k) B. Léger, A. Denicourt-Nowicki, H. OlivierBourbigou and A. Roucoux, Inorg. Chem., 2007, 47, 90909096; (I) A. Denicourt-Nowicki, B. Léger and A. Roucoux, Phys. Chem. Chem. Phys., 2011, 13, 13510-13517; (m) R. R. Dykeman, N. Yan, R. Scopelliti and P. J. Dyson, Inorg. Chem., 2011, 50, 717-719. Hydroxyl: (n) N. Yan, X. Yang, Z. Fei, Y. Li, Y. Kou and P. J. Dyson, Organometallics, 2009, 28, 937-939; (o) X. Yuan, N. Yan, S. A. Katsyuba, E. E. Svereva, Y. Kou and P. J. Dyson, Phys. Chem. Chem. Phys., 2012, 14, 6026-6033. Phosphine: (p) B. C. Leal, C. S. Consorti, G. Machado and J. Dupont, Catal. Sci. Technol., 2015, 5, 903-909; (q) K. L. Luska and A. Moores, Adv. Synth. Catal., 2011, 353, 3167-3177; (r) S. Bahadorikhalili, L. Ma'mani, H. Mahdavi and A. Shafiee, RSC Adv., 2015, 5, 71297-71305.

27 (a) S. Doherty, J. G. Knight, J. R. Ellison, D. Weekes, R. W. Harrington, C. Hardacre, and H. Manyar, Green Chem., 2012, 14, 925-929; (b) S. Doherty, J. G. Knight, M. A. Carroll, J. R. Ellison, S. J. Hobson, S. Stevens, C. Hardacre and P. Goodrich, Green Chem., 2015, 17, 1559-1572; (c) S. Doherty, J. G. Knight, M. A. Carroll, A. R. Clemmet, R. R. Ellison, T. Backhouse, N. Holmes, L. A. Thompson and R. A Bourne, RSC Adv., 2016, 6, 73118-73131; (d) S. Doherty, J. G. Knight, J. R. Ellison, P. Goodrich, L. Hall, C. Hardacre, M. J. Muldoon, S. Park, A. Ribeiro, C. A. N. de Castro, M. J. Lourenco and P. Davy, Green Chem., 2014, 16, 1470-1479.

28 (a) S. G. Kwon, G. Krylova, A. Sumer, M. M. Schwartz, E. E. Bunel, C. L. Marshall, S. Chattopadhyay, B. Lee, J. Jellinek and E. V. Shevchenko, Nano Lett., 2012, 12, 5382-5388; (b) Z. Guo, C. Xiao, R. V. Maligal-Ganesh, L. Zhou, T. W. Goh, X. Li, D. Tesfagaber, A. Thiel and W. Huang, ACS Catal., 2014, 4 1340-1348; (c) K. R. Kahsar, D. K. Schwartz and J. W. Medlin, J. Am. Chem. Soc., 2014, 136, 520-526. (d) I. Cano, A. M. Chapman, A. Urakawa and P. W. N. M. van Leeuwen, J. Am. Chem. Soc., 2014, 136, 2520-2528.

29 S. Doherty, J. G. Knight, T. Backhouse, E. Abood, H. Alshaikh, I. J. S. Fairlamb, R. A. Bourne, T. W. Chamberlain and R. Stones, Green Chem., 2017, 19, 1635-1641.

30 (a) T. Iwai, T. Harada, K. Hara and M. Sawamura, Angew. Chem. Int. Ed., 2013, 52, 12322-12326; (b) Y.-B. Zhou, C.-Y. Li, M. Lin, Y.-J. Ding, Z.-P. Zhan, Adv. Synth. Catal., 2015, 357 2503-2508.

31 J.-K. Sun, Z. Kochovski, W.-Y. Zhang, H. Kirmse, Y. Lu, M Antonietti and J. Yuan, J. Am. Chem. Soc. 2017, 139, 89718976.

32 Y. Wang, H. Zhong, L. Li and R. Wang, ChemCatChem, 2016 , 8, 2234-2240.

33 A. Pourjavadi, N. Safaie, S. H. Hosseini and C. Bennett, J. Ind. Eng. Chem., 2016, 38, 82-92.

34 J. Li, X.-Y. Shi, Y.-Y. Bi, J.-F. Wei and Z.-G. Chen, ACS Catal., 2011, 1, 657-664.

35 N. J. Costa, P. K. Kiyohara, A. L. Monteirro, Y. Coppel, K. Philippot and L. M. Rossi, J. Catal., 2010, 276, 382-389.

36 K. Sawai, R. Tatumi, T. Nakahodo and H. Fujihara, Angew. Chem. Int. Ed., 2008, 47, 6917-6919. 
37 M. Chatterjee, A. Chatterjee, H. Kawanami, T. Ishizaka, T. Suzuki and A. Suzuki, Adv. Synth. Catal., 2012, 354, 20092018.

38 H. Zhao, Y. Wang and R. Wang, Chem Commun., 2014, 50 10871-10873.

39 D. Nandi, S. Siwal, M. Choudhary and K. Mallick, Appl. Catal. A: General, 2016, 523, 31-38.

40 L. R. S. Lara, A. D. Zottis, W. C. Elias, D. Faggion Jr, C. E. Maduro de Campos, J. S. Acuña and J. B. Domingos, RSC Adv. 2015, 5, 8289-8296.

41 C. Wang, R. Ciganda, L. Salmon, D. Gregurec, J. Irigoyen, S. Moya, J. Ruiz, D. Gregurec, J. Irigoyen, S. Moya, F. Ruiz, D. Astruc, Angew. Chem. Int. Ed., 2016, 55, 3091-3095.

42 (a) E. A. Gelder, S. D. Jackson and C. M. Lok, Chem. Commun., 2005, 4, 522-524; (b) A. Corma, P. Concepcion and P. Serena, Angew. Chem Int. Ed., 2007, 46, 7266-7269; (c) L. Pernoud, J. P. Candy, B. Didillon, R. Jacquot and J. M. Basset, Stud. Surf. Sci. Catal., 2000, 130, 2057-2062; (d) S. Zhang, C. R. Chang, Z. Q. Huang, J. Ii, Z. Wu. Y. Ma, Z. Zhang, Y. Wang, and Y. Qu, J. Am. Chem. Soc. 2016, 138, 2629-2637.

43 M. Turáková, T. Salmi, K. Eränen, J. Wårnä, D. Y. Murzin and M. Králik, Appl. Catal., A, 2015, 499, 66-76.

44 J. Sun, Y. Fu, G. He, X. Sun and X. Wang, Cat. Sci. \& Technol., 2014, 4, 1742-1748.

45 K. N. Mochalov; V. S. Khain; G. G. Gil'manshin KInetika I Kataliz 1965, 6, 541.

46 A. Kumar, K. Purkait, S. Kr. Dey, A. Sarkar and A. Mukherjee, RSC Adv., 2014, 4, 35233-35237.

47 (a) S. Jayakumar, A. Modak, M. Guo, H. Li, X. Hu and Q. Yang, Chem. Eur. J., 2017, 23, 7791-7797. For other examples of ligand effects in NP catalysis see: (b) J. L. Castelbou, A. Gual, E. Mercadé, C. Claver and C. Godard, Catal. Sci. Technol., 2013, 3, 2828-2833; (c) D. González-Gálvez, P. Nolid, K. Philippot, B. Chaudret and P. W. M. N. van Leewuen, ACS Catal., 2012, 2, 317-321; (d) D. González-Gálvez, P. Lara, O. Rivada-Wheelaghan, S. Conejero, B. Chaudret, K. Philippot and P. W. N. M. van Leewuen, Catal. Sci. Technol., 2013, 3 99-105; (e) J. B. Ernst, C. Schwermann, G. Yokota, M. Tada, S Muratsugu, N. L. Doltsinis and F. Glorious, J. Am. Chem. Soc. 2017, 139, 9144-9147; (f) J. B, Ernst, S. Muratsugu, F. Wang M. Tada and F. Glorius, J. Am. Chem. Soc., 2016, 138, 10718 10721; (g) C. Richter, K. Schaepe, F. Glorious and B. J. Ravoo, Chem. Commun.; 2014, 50, 3204-3207; (h) A. Ferry, K. Schaepe, P. Tegeder, C. Richter, K. M. Chepiga, B. J. Ravoo and F. Glorius, ACS Catal.; 2015, 5, 5414-5420; (i) A. Rühling, K. Schaepe, L. Rakers, B. Vonhoren, P. Tegeder, B. J. Ravoo and F. Glorious, Angew. Chem. Int. Ed., 2016, 55, 5856-5860. 